\title{
On Catalan Trees and the Jacobian Conjecture
}

\author{
Dan Singer \\ Oakland University \\ Rochester, MI \\ dwsinger@oakland.edu
}

Submitted: July 11, 2000; Accepted: November 28, 2000

\begin{abstract}
New combinatorial properties of Catalan trees are established and used to prove a number of algebraic results related to the Jacobian conjecture. Let $F=\left(x_{1}+H_{1}, x_{2}+H_{2}, \ldots, x_{n}+H_{n}\right)$ be a system of $n$ polynomials in $C\left[x_{1}, x_{2}, \ldots, x_{n}\right]$, the ring of polynomials in the variables $x_{1}, x_{2}, \ldots, x_{n}$ over the field of complex numbers. Let $H=\left(H_{1}, H_{2}, \ldots, H_{n}\right)$. Our principal algebraic result is that if the Jacobian of $F$ is equal to 1 , the polynomials $H_{i}$ are each homogeneous of total degree 2 , and $\left(\frac{\partial H_{i}}{\partial x_{j}}\right)^{3}=0$, then $H \circ H \circ H=0$ and $F$ has an inverse of the form $G=\left(G_{1}, G_{2}, \ldots, G_{n}\right)$, where each $G_{i}$ is a polynomial of total degree $\leq 6$. We prove this by showing that the sum of weights of Catalan trees over certain equivalence classes is equal to zero. We also show that if all of the polynomials $H_{i}$ are homogeneous of the same total degree $d \geq 2$ and $\left(\frac{\partial H_{i}}{\partial x_{j}}\right)^{2}=0$, then $H \circ H=0$ and the inverse of $F$ is $G=\left(x_{1}-H_{1}, \ldots, x_{n}-H_{n}\right)$.
\end{abstract}

\section{Introduction}

Let $F_{1}, F_{2}, \ldots, F_{n}$ be polynomials in $C\left[x_{1}, x_{2}, \ldots, x_{n}\right]$, the ring of polynomials in the variables $x_{1}, x_{2}, \ldots, x_{n}$ over the field of complex numbers. The Jacobian conjecture states that if the Jacobian of the system $F=\left(F_{1}, F_{2}, \ldots, F_{n}\right)$ is equal to a non-zero scalar number, then there exists an inverse system of polynomials $G=\left(G_{1}, G_{2}, \ldots, G_{n}\right)$ such that

$$
G_{i}\left(F_{1}, F_{2}, \ldots, F_{n}\right)=x_{i}
$$

for each $i \leq n$. For example, let $n=2$ and consider

$$
F_{1}=x_{1}+\left(x_{1}+x_{2}\right)^{2}, \quad F_{2}=x_{2}-\left(x_{1}+x_{2}\right)^{2} .
$$

Keywords: Catalan trees, Jacobian conjecture, formal tree expansions AMS Subject Classifications: 05E99 (primary), 05A99, 05C05, 14R15 (secondary) 
Since

$$
F_{1}-\left(F_{1}+F_{2}\right)^{2}=x_{1}
$$

and

$$
F_{2}+\left(F_{1}+F_{2}\right)^{2}=x_{2},
$$

the inverse to the system $F=\left(F_{1}, F_{2}\right)$ is the system $G=\left(G_{1}, G_{2}\right)$ defined by

$$
G_{1}=x_{1}-\left(x_{1}+x_{2}\right)^{2}, \quad G_{2}=x_{2}+\left(x_{1}+x_{2}\right)^{2} .
$$

Note that the Jacobian of $F$ is

$$
\operatorname{det}\left[\begin{array}{cc}
\frac{\partial F_{1}}{\partial x_{1}} & \frac{\partial F_{1}}{\partial x_{2}} \\
\frac{\partial F_{2}}{\partial x_{1}} & \frac{\partial F_{2}}{\partial x_{2}}
\end{array}\right]=\operatorname{det}\left[\begin{array}{cc}
1+2 x_{1}+2 x_{2} & 2 x_{1}+2 x_{2} \\
-2 x_{1}-2 x_{2} & 1-2 x_{1}-2 x_{2}
\end{array}\right]=1 .
$$

There are a number of partial results relating to systems in which $F_{i}=$ $x_{i}+H_{i}$ for all $i$, where each $H_{i}$ is homogeneous of the same total degree $d$. In this case the matrix of partial derivatives $\left(\frac{\partial H_{i}}{\partial x_{j}}\right)$ satisfies $\left(\frac{\partial H_{i}}{\partial x_{j}}\right)^{n}=0$. Wang [4] and Oda [3] have shown that the Jacobian conjecture is true of those systems for which $d=2$. Bass, Connell and Wright [1] have shown that the Jacobian conjecture is true provided it is true of all systems for which $d=3$. A number of authors have shown (see for example [2]) that the Jacobian conjecture is true when $\left(\frac{\partial H_{i}}{\partial x_{j}}\right)^{2}=0$, and in this case the inverse system is given by $G_{i}=x_{i}-H_{i}$ for each $i$. David Wright [5] gave a combinatorial proof of this result when $n=2$ and $d=3$, using the formal tree expansion of the inverse suggested by Gurjar's formula (unpublished, but cited in [5]). While Wright's formal tree expansion is an elegant combinatorial expression of the inverse, his tree surgery approach does not easily lend itself to calculating the terms in the differential ideal generated by $\left(\frac{\partial H_{i}}{\partial x_{j}}\right)^{n}$. In this paper we propose a different approach to the formal tree expansion of the inverse, and our methods give rise to the following algebraic results:

Theorem 1.1. Let $F=\left(x_{1}+H_{1}, x_{2}+H_{2}, \ldots, x_{n}+H_{n}\right)$ be a system of polynomials with complex coefficients, where each $H_{i}$ is homogeneous of total degree $d$. Let $H=\left(H_{1}, H_{2}, \ldots, H_{n}\right)$. If $\left(\frac{\partial H_{i}}{\partial x_{j}}\right)^{2}=0$ then the inverse of $F$ is $\left(x_{1}-H_{1}, x_{2}-H_{2}, \ldots, x_{n}-H_{n}\right)$ and $H \circ H=0$, regarding $H$ as a function from polynomial systems to polynomial systems. If $\left(\frac{\partial H_{i}}{\partial x_{j}}\right)^{3}=0$ and $d=2$ then $F$ has a polynomial inverse of degree $\leq 6$ and $H \circ H \circ H=0$.

We should remark that Bass, Connell and Wright [1] proved that $2^{n-1}$ is a bound on the degree of the inverse of $F$ when $F$ is a quadratic system of $n$ polynomials in $n$ variables. Our bound on the degree of the inverse is much lower than this for large $n$, given our additional hypothesis that $\left(\frac{\partial H_{i}}{\partial x_{j}}\right)^{3}=0$.

As an illustration of the property that $\left(\frac{\partial H_{i}}{\partial x_{j}}\right)^{2}=0 \Rightarrow H \circ H=0$, consider our initial example. In this case we have

$$
H_{1}=\left(x_{1}+x_{2}\right)^{2}, \quad H_{2}=-\left(x_{1}+x_{2}\right)^{2},
$$




$$
\left[\begin{array}{ll}
\frac{\partial H_{1}}{\partial x_{1}} & \frac{\partial H_{1}}{\partial x_{2}} \\
\frac{\partial H_{2}}{\partial x_{1}} & \frac{\partial H_{2}}{\partial x_{2}}
\end{array}\right]^{2}=\left[\begin{array}{cc}
2 x_{1}+2 x_{2} & 2 x_{1}+2 x_{2} \\
-2 x_{1}-2 x_{2} & -2 x_{1}-2 x_{2}
\end{array}\right]^{2}=\left[\begin{array}{ll}
0 & 0 \\
0 & 0
\end{array}\right],
$$

and

$$
H \circ H=\left(H_{1}\left(H_{1}, H_{2}\right), H_{2}\left(H_{1}, H_{2}\right)\right)=\left(\left(H_{1}+H_{2}\right)^{2},-\left(H_{1}+H_{2}\right)^{2}\right)=(0,0) .
$$

This paper is organized as follows. In Section 2 we show that the formal power series inverse of a system of polynomials can be expressed as sums of weights of Catalan trees. In Section 3 we will indicate how a combinatorial interpretation of $\left(\frac{\partial H_{i}}{\partial x_{j}}\right)^{n}=0$ can be combined with Gaussian elimination to show that sums of weights over equivalence classes of Catalan trees having a sufficiently large number of external vertices are zero. In order to obtain this result we will need to establish new combinatorial properties of Catalan trees. This is the subject of Section 4. In Section 5 we use our understanding of Catalan trees to prove Theorem 1.1. Our methods give rise to a number of difficult questions about these combinatorial objects, which we pose in the concluding section of this paper.

\section{Catalan Tree Expansion of the Inverse}

Catalan trees are rooted planar trees whose internal vertices have out-degree $\geq 2$. We will denote the set of Catalan trees by $\mathcal{C}$ and the set of Catalan trees having $p$ external vertices by $\mathcal{C}_{p}$. Internal vertices are vertices which have successor vertices, and external vertices are those which do not (they are also known as leaves). For example, $\mathcal{C}_{4}$ consists of the trees

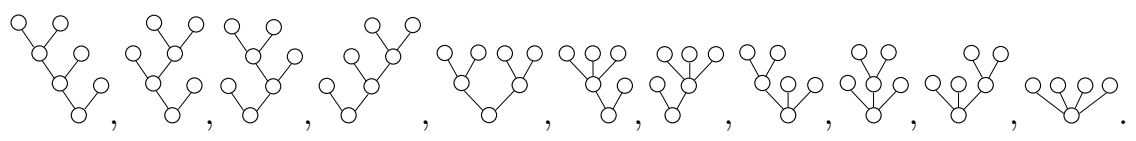

By definition, for $p \geq 2$ we have

$$
\mathcal{C}_{p}=\bigcup_{\substack{2 \leq k \leq p \\ p_{1}+\cdots+p_{k}=p}}\{\underbrace{T_{1} T_{2} \cdots T_{k}}: T_{1} \in \mathcal{C}_{p_{1}}, \ldots, T_{k} \in \mathcal{C}_{p_{k}}\} .
$$

In order to express the inverse of $F=x+H$ as sums of weights of Catalan trees, we need to introduce the notion of vertex colors. Given the finite set of 
colors $\{1,2, \ldots, n\}$, we recursively define for each $i \leq n$ the set $\mathcal{C}^{(i)}$, consisting of colored Catalan trees with root colored $i$, by

$$
\mathcal{C}^{(i)}=\bigcup_{p=1}^{\infty} \mathcal{C}_{p}^{(i)},
$$

where

$$
\mathcal{C}_{1}^{(i)}=\left\{\bigcirc_{i}\right.
$$

and

$$
\begin{aligned}
& \mathcal{C}_{p}^{(i)}= \\
& \bigcup \\
& 2 \leq k \leq p \\
& p_{1}+\cdots+p_{k}=p \\
& 1 \leq i_{1} \leq \cdots \leq i_{k} \leq n \\
& T_{1} T_{2} \cdots T_{k} \\
& \left\{\mathcal{O}_{i}: T_{1} \in \mathcal{C}_{p_{1}}^{\left(i_{1}\right)}, \ldots, T_{k} \in \mathcal{C}_{p_{k}}^{\left(i_{k}\right)}\right\} .
\end{aligned}
$$

Figure 2.1 contains an illustration of a colored tree in $\mathcal{C}_{7}^{(1)}$.

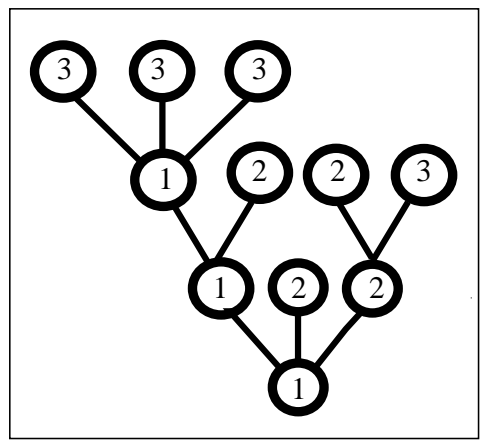

Figure 2.1: A Colored Tree

Given a system of polynomials $F=\left(F_{1}, F_{2}, \ldots, F_{n}\right)$, where $F_{i}=x_{i}+H_{i}$ and

$$
\begin{aligned}
& H_{i}=\sum_{k \geq 2} \\
& 1 \leq i_{1} \leq i_{2} \leq \cdots \leq i_{k} \leq n \\
& h_{i_{1}, i_{2}, \ldots, i_{k}}^{(i)} x_{i_{1}} x_{i_{2}} \cdots x_{i_{k}},
\end{aligned}
$$

we define a weight function $w$ on $\bigcup_{i=1}^{n} \mathcal{C}^{(i)}$ in the following way: Let $T \in \mathcal{C}^{(i)}$. Let $V_{I}(T)$ denote the set of internal vertices of $T$, and let $V_{E}(T)$ denote the set of external vertices of $T$. For each vertex $v$ of $T$, let $c(v)$ denote the color of $v$. 
For each internal vertex $v$ of $T$, let $m(v)$ denote the multiset consisting of the colors of the immediate successors of $v$. We then define

$$
w(T)=(-1)^{\left|V_{I}(T)\right|} \prod_{v \in V_{I}(T)} h_{m(v)}^{(c(v))} \prod_{v \in V_{E}(T)} x_{c(v)} .
$$

For example, the weight of the colored tree in Figure 2.1 is

$$
h_{1,2,2}^{(1)} h_{1,2}^{(1)} h_{3,3,3}^{(1)} h_{2,3}^{(2)} x_{2}^{3} x_{3}^{4} .
$$

An alternate way to compute the weight function is by means of the recursive definition

$$
\begin{aligned}
w\left(\mathrm{O}_{i}\right) & =x_{i}, \\
w\left(\mathrm{O}_{i}^{T_{2}}\right) & =-h_{i_{1}, i_{2}, \ldots, i_{k}}^{(i)} \prod_{j=1}^{k} w\left(T_{j}\right),
\end{aligned}
$$

where $T_{j} \in \mathcal{C}^{\left(i_{j}\right)}$ for each $j$.

We can now express the formal power series inverse of the system $F$ as sums of weights of Catalan trees. We define $G_{i} \in C\left[\left[x_{1}, x_{2}, \ldots, x_{n}\right]\right]$ for each $i$ by

$$
G_{i}=\sum_{T \in \mathcal{C}^{(i)}} w(T)
$$

This sum is well-defined because the total degree of $w(T)$ is $p$ for all $T \in \mathcal{C}_{p}^{(i)}$, and each of the sets $\mathcal{C}_{p}^{(i)}$ is finite.

Theorem 2.1. With notation as above,

$$
F_{i}\left(G_{1}, G_{2}, \ldots, G_{n}\right)=x_{i}
$$

for each $i$. 
Proof. Using the definition of $\mathcal{C}^{(i)}$ and the recursive definition of the weight function, we have

$$
\begin{aligned}
& G_{i}=\sum_{T \in \mathcal{C}^{(i)}} w(T) \\
& =x_{i}+\sum_{k \geq 2} \\
& 1 \leq i_{1} \leq i_{2} \leq \cdots \leq i_{k} \leq n \\
& T_{1} \in \mathcal{C}^{\left(i_{1}\right)}, \ldots, T_{k} \in \mathcal{C}^{\left(i_{k}\right)} \\
& =x_{i}-\quad \sum_{k \geq 2} \quad h_{i_{1}, i_{2}, \ldots, i_{k}}^{(i)} \prod_{j=1}^{k} w\left(T_{j}\right) \\
& 1 \leq i_{1} \leq i_{2} \leq \cdots \leq i_{k} \leq n \\
& T_{1} \in \mathcal{C}^{\left(i_{1}\right)}, \ldots, T_{k} \in \mathcal{C}^{\left(i_{k}\right)} \\
& =x_{i}-\sum_{k \geq 2} \quad h_{i_{1}, i_{2}, \ldots, i_{k}}^{(i)} G_{i_{1}} G_{i_{2}} \cdots G_{i_{k}} \\
& 1 \leq i_{1} \leq i_{2} \leq \cdots \leq i_{k} \leq n \\
& =x_{i}-H_{i}\left(G_{1}, G_{2}, \ldots, G_{n}\right),
\end{aligned}
$$

hence

$$
F_{i}\left(G_{1}, G_{2}, \ldots, G_{n}\right)=G_{i}+H_{i}\left(G_{1}, G_{2}, \ldots, G_{n}\right)=x_{i}
$$

for each $i$.

It will be convenient to ignore the vertex colors of a tree $T \in \mathcal{C}^{(i)}$ and to regard only the underlying tree, shape $(T)$, which resides in $\mathcal{C}$. This leads us to define the weight function $w_{i}$ on $\mathcal{C}$ by

$$
w_{i}(T)=\sum_{\begin{array}{c}
S \in \mathcal{C}^{(i)} \\
\operatorname{shape}(S)=T
\end{array}} w(S) .
$$

Using this definition we have

$$
G_{i}=\sum_{T \in \mathcal{C}} w_{i}(T)
$$

The Jacobian conjecture states that if the Jacobian of $F=\left(F_{1}, F_{2}, \ldots, F_{n}\right)$ is a non-zero scalar, then each $G_{i}$ is a polynomial. This is equivalent to saying that

$$
\sum_{T \in \mathcal{C}_{p}} w_{i}(T)=0
$$


for all $i$ and sufficiently large $p$. In the next section, we will use a combinatorial argument to prove that if $\left(\frac{\partial H_{i}}{\partial x_{j}}\right)^{2}=0$ and each $H_{i}$ is homogeneous of degree 2 then $H \circ H=0$ is true, and we will describe a strategy for proving 2.1. This will motivate the subsequent combinatorial analysis of Catalan trees.

\section{$3 \quad$ Exploiting $\left(\frac{\partial H_{i}}{\partial x_{j}}\right)^{n}=0$}

If $F=\left(x_{1}+H_{1}, x_{2}+H_{2}, \ldots, x_{n}+H_{n}\right)$ is a system of polynomials having Jacobian equal to 1 , and if each $H_{i}$ is homogeneous of the same total degree, then $\left(\frac{\partial H_{i}}{\partial x_{j}}\right)^{n}=0$. We can translate this fact into a combinatorial property of a certain class of Catalan trees. We will begin by defining marked Catalan trees and the formal multiplication of marked trees with other Catalan trees.

A marked Catalan tree is a pair $(T, v)$, where $T$ is a Catalan tree and $v$ is an external vertex of $T$. We will denote by $(\mathcal{C}, *)$ the set of marked Catalan trees. Marked Catalan trees having $p$ external vertices are denoted by $\left(\mathcal{C}_{p}, *\right)$, marked colored Catalan trees with root colored $i$ are denoted by $\left(\mathcal{C}^{(i)}, *\right)$, etc. We will also denote by $\mathcal{C}^{(i, j)}$ the set $\left\{(T, v) \in\left(\mathcal{C}^{(i)}, *\right): c(v)=j\right\}$, where $c(v)$ denotes the color of the vertex $v$. The shape of a marked Catalan tree is the underlying marked Catalan tree (minus the vertex colors, but including the same marked vertex).

Marked Catalan trees can be multiplied together in a natural way. Let $(S, u)$ and $(T, v)$ be elements of $(\mathcal{C}, *)$. We set $(S, u)(T, v)$ equal to the marked tree obtained by replacing the vertex $u$ in $S$ by $(T, v)$. For example, if

$$
(S, u)=\text { of }
$$

and

$$
(T, v)=\wp
$$

then

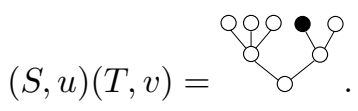

Similarly, we can multiply a marked tree $(S, u)$ and an unmarked tree $T$ to obtain an unmarked tree $(S, u) T$.

We will extend our weight function to marked Catalan trees as follows:

$$
w_{i, j}(T, v)=(-1)^{\left|V_{I}(T)\right|} \sum_{\substack{(S, v) \in \mathcal{C}^{(i, j)} \\ \operatorname{shape}(S, v)=(T, v)}} \prod_{u \in V_{I}(S)} h_{m(u)}^{(c(u))} \prod_{u \in V_{E}(S)-\{v\}} x_{c(u)}
$$




$$
=\frac{1}{x_{j}} \sum_{\substack{(S, v) \in \mathcal{C}^{(i, j)} \\ \operatorname{shape}(S, v)=(T, v)}} w(S) .
$$

Note that with this definition we have

$$
w_{i, j}((S, u)(T, v))=\sum_{k=1}^{n} w_{i, k}(S, u) w_{k, j}(T, v)
$$

and

$$
w_{i}((S, u) T)=\sum_{j=1}^{n} w_{i, j}(S, u) w_{j}(T)
$$

Of particular interest are those marked trees having height equal to the number of their internal vertices, which we call chains. For example, the marked tree

$$
(T, v)=90^{0}
$$

is a chain of height 3 . We will denote the set of all chains in $(\mathcal{C}, *)$ by $C H$ and those of height $k$ by $C H_{k}$. Note that a chain of height $k$ can be viewed as the formal product of $k$ chains of height 1 . With notation as in Section 2, we have

$$
\sum_{(T, v) \in C H_{1}} w_{i, j}(T, v)=-\frac{\partial H_{i}}{\partial x_{j}}
$$

Therefore we have the matrix identity

$$
\left(\sum_{(T, v) \in C H_{k}} w_{i, j}(T, v)\right)=\left(\sum_{(T, v) \in C H_{1}} w_{i, j}(T, v)\right)^{k}=(-1)^{k}\left(\frac{\partial H_{i}}{\partial x_{j}}\right)^{k}
$$

for each positive integer $k$. In particular, we have the following theorem:

Theorem 3.1. With notation as above, if $F=\left(x_{1}+H_{1}, x_{2}+H_{2}, \ldots, x_{n}+H_{n}\right)$ is a system of polynomials with Jacobian equal to 1 , and if each $H_{i}$ is homogeneous of the same total degree, then

$$
\left(\sum_{(T, v) \in C H_{n}} w_{i, j}(T, v)\right)=(-1)^{n}\left(\frac{\partial H_{i}}{\partial x_{j}}\right)^{n}=0 .
$$

In combinatorics, a picture is worth a thousand definitions. Keeping this in mind, we will give a combinatorial argument that $H \circ H=0$, given that $H=$ $\left(H_{1}, H_{2}, \ldots, H_{n}\right)$ is a system of polynomials such that each $H_{i}$ is homogeneous 
of degree 2 and that $\left(\frac{\partial H_{i}}{\partial x_{j}}\right)^{2}=0$. This will motivate the definitions to come when we make our more general arguments.

Given a Catalan tree $T$, we will let $[T]$ denote the equivalence class of all trees isomorphic to $T$ as a rooted tree. Given a marked Catalan tree $(T, v)$, we will let $[T, v]$ denote the equivalence class of all trees isomorphic to $(T, v)$ as a rooted tree, where the isomorphism sends marked vertex to marked vertex. For example, the trees isomorphic to

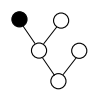

are

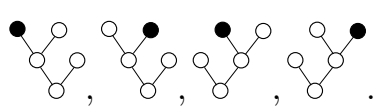

We will denote by $w_{i}[T]$ and $w_{i, j}[T, v]$ the expressions

$$
w_{i}[T]=\sum_{S \in[T]} w_{i}(T)
$$

and

$$
w_{i, j}[T, v]=\sum_{(S, v) \in[T, v]} w_{i, j}(S, v) .
$$

In order to show that $H \circ H=0$, we must show that

$$
w_{i}\left[\mathfrak{g}_{0}^{0}\right]=0
$$

for each $i$. We know that

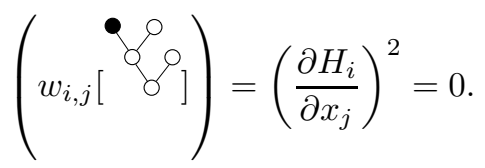

Let $p$ and $q$ be indeterminants. Regarding

$$
w_{i, j}\left[\begin{array}{l}
0 \\
0
\end{array}\right]
$$

as a function of $x_{1}, x_{2}, \ldots, x_{n}$, we have

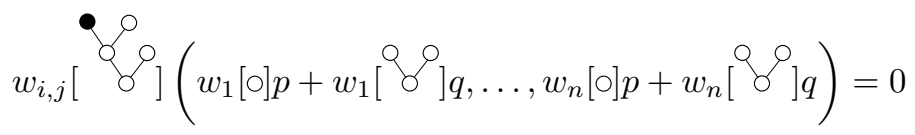

for each $i$ and $j$. On the other hand,

$$
w_{i, j}\left[\oint_{\delta}^{\circ}\right]\left(w_{1}[\circ] p+w_{1}\left[\delta^{\circ}\right] q, \ldots, w_{n}[\circ] p+w_{n}\left[\zeta^{\circ}\right] q\right)=
$$

THE ELECTRonic JOURnAl of COMBINATORICs 8 (2001), \#R2 


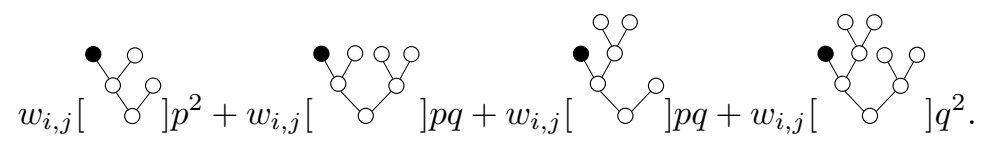

Hence, taking the coefficient of $p q$, we obtain

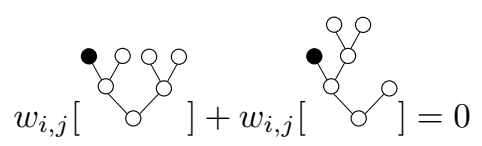

for each $i$ and $j$.

Observe that we have the matrix equation

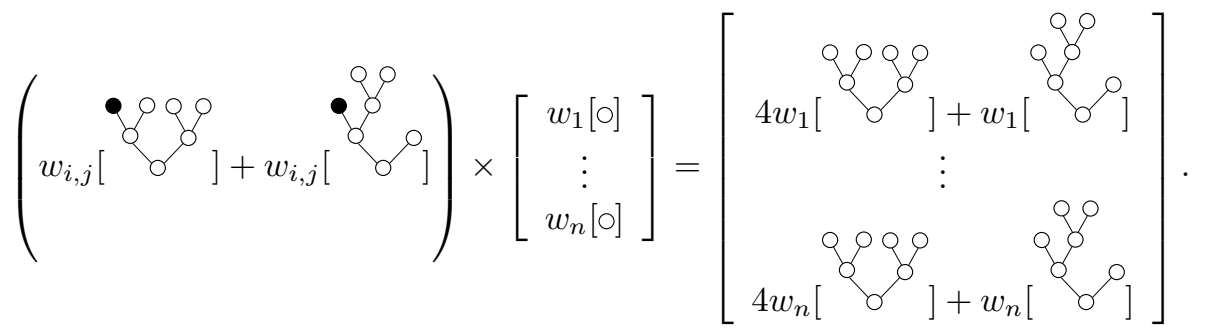

The multiplicity 4 arises because there are four ways to produce

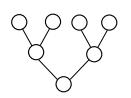

by multiplying an element in the class of

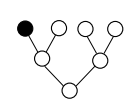

and an element in the class of $\circ$. We can now say that

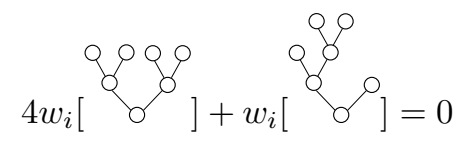

for each $i \leq n$. On the other hand, we also have

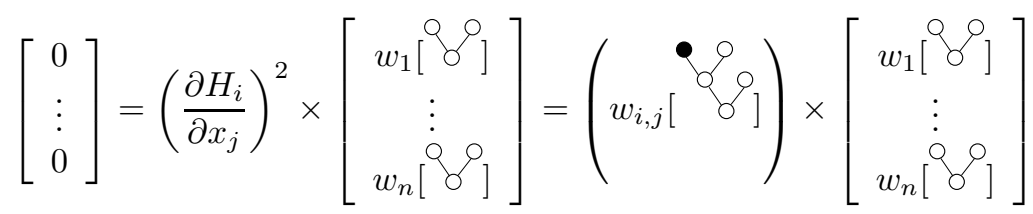




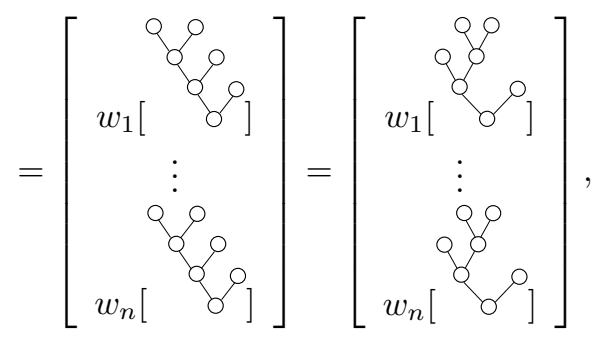

the last equality holding because we are summing over an equivalence class of trees. Hence

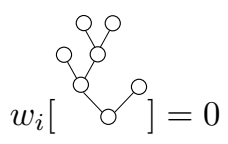

for each $i$. Combining equations 3.2 and 3.3 we arrive at 3.1. The multiplicity 4 encountered in 3.2 illustrates why we need to work in a field of characteristic zero.

The arguments leading to 3.1 are rather ad hoc. However, our strategy is clear: in order to prove that $w_{i}[T]=0$ for some Catalan tree $T$, we must identify a finite subset of trees $\left\{T_{1}, \ldots, T_{k}\right\}$ which contains $T$, produce a collection $L$ of linear combinations of the form

$$
\sum_{j} \alpha_{j} w_{i}\left[T_{j}\right]
$$

show that $w_{i}[T]$ belongs to the span of the elements of $L$ over the rationals by performing Gaussian elimination, and show that each element of $L$ evaluates to zero when $\left(\frac{\partial H_{i}}{\partial x_{j}}\right)^{n}=0$. In order to perform Gaussian elimination, one must impose an ordering on $\left\{T_{1}, \ldots, T_{k}\right\}$ and characterize the leading term of each element of $L$. This is the focus of the remainder of this paper.

\section{Combinatorial Properties of Catalan Trees}

\section{Equivalence Classes of Catalan Trees}

We begin by defining carefully the equivalence relation on $\mathcal{C}$. Two trees are equivalent if and only if they are isomorphic as rooted non-planar graphs. Hence equivalent trees must have the same number of external vertices. In general, if $S$ and $T$ are trees with at least two external vertices, and

$$
S=\underbrace{S_{1} S_{2} \cdots S_{j}}
$$

and

$$
T=\underbrace{T_{1} T_{2} \ldots T_{k}}
$$


then $S$ is equivalent to $T$ if and only if $j=k$ and there exists a permutation $\sigma$ such that $T_{i} \equiv S_{\sigma(i)}$ for all $i$. We will define an equivalence relation on $(\mathcal{C}, *)$ similarly, adding that the graph isomorphism must map a marked vertex to another marked vertex. No marked tree is equivalent to an unmarked tree.

\section{Branch Words, Multisets, Chains, and Shuffles}

In order to exploit $\left(\frac{\partial H_{i}}{\partial x_{j}}\right)^{n}=0$, we must have a language to describe the chains which occur in each Catalan tree. We define the branch word $B_{v}(T)$ of a tree $(T, v) \in(\mathcal{C}, *)$ recursively as follows. If $(T, v)$ consists of a single marked vertex then we set $B_{v}(T)$ equal to the empty word. Otherwise, write

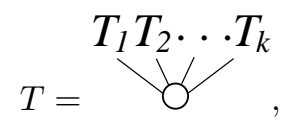

and suppose that $v \in V_{E}\left(T_{i}\right)$. Let $M$ represent the multiset of subtrees $\left\{T_{j}\right.$ : $1 \leq j \leq k$ and $j \neq i\}$. We set $B_{v}(T)$ equal to the word $B_{v}\left(T_{i}\right) M$. We say that branch words $B_{1}=M_{1} M_{2} \ldots M_{j}$ and $B_{2}=N_{1} N_{2} \ldots N_{k}$ are equivalent to each other if and only if $j=k$ and $M_{i} \equiv N_{i}$ for all $i$, that is if there is a bijection $\phi_{i}: M_{i} \rightarrow N_{i}$ for each $i$ such that $T \equiv \phi_{i}(T)$ for all $T \in M_{i}$.

The branch multiset $M_{v}(T)$ of a marked tree $(T, v)$ is the union of the multisets occuring in the branch word $B_{v}(T) . M_{v}(T)$ contains the subtrees branching from the unique path in $T$ from the root to $v$. The subtree of $T$ at a vertex $u$ is defined to be the induced subgraph of $T$ on the vertex $u$ and all of its successors in $T$.

The chain $C_{v}(T)$ of a marked tree $(T, v)$ is the marked tree that results by replacing each of the subtrees of $T$ in $M_{v}(T)$ by the tree with a single vertex.

A shuffle of a marked tree $(T, v)$ is any marked tree $\left(T^{\prime}, v\right)$ that results by replacing the external vertices of the chain $C_{v}(T)$ by the subtrees of $T$ in $M_{v}(T)$ in something other than their original positions in $T$. A shuffle of an unmarked tree $T$ is any tree $T^{\prime}$ that results by factoring $T$ into $(A, u)(B, v) C$, shuffling $(B, v)$ to obtain $\left(B^{\prime}, v\right)$, and setting $T^{\prime}$ equal to $(A, u)\left(B^{\prime}, v\right) C$.

As an illustration of these ideas, let

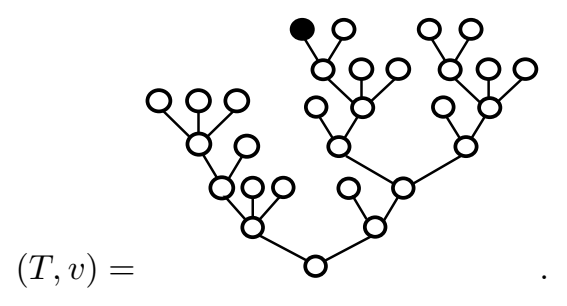


Then

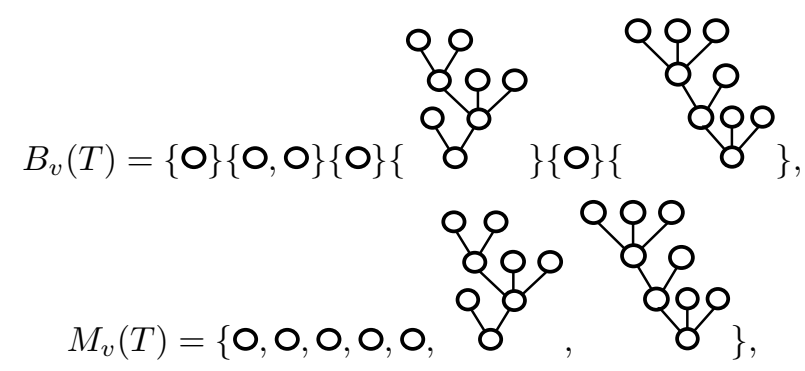

and

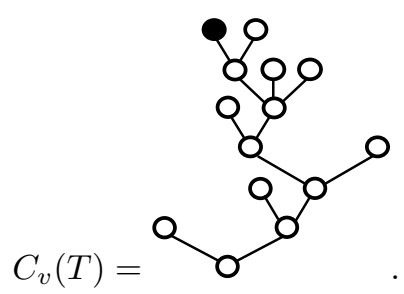

One possible shuffle of $(T, v)$ is

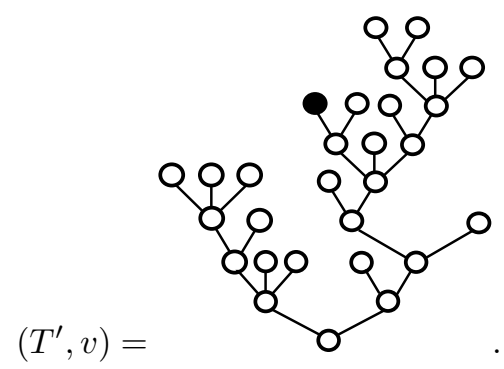

The following lemma shows that equivalent marked trees have equivalent branch words.

Lemma 4.1. Let $(S, u),(T, v) \in(\mathcal{C}, *)$. Then $(S, u) \equiv(T, v)$ if and only if $B_{u}(S) \equiv B_{v}(T)$.

Proof. Suppose $(S, u) \equiv(T, v)$. Then $S, T \in \mathcal{C}_{p}$ for some $p$. We will prove the conclusion by induction on $p$. If $p=1$ then $B_{u}(S)$ and $B_{v}(T)$ are both equal to the empty word. Now consider $p>1$. Write

$$
S=S^{S_{1} S_{2} \cdots S_{k}}
$$

and

$$
T=\underbrace{T_{1} T_{2} \ldots T_{k}}
$$


for some $k \geq 2$. Suppose $u \in V_{E}\left(S_{i_{0}}\right)$. Then there exists a permutation $\sigma$ such that $S_{i} \equiv T_{\sigma(i)}$ for all $i \neq i_{0}$ and $\left(S_{i_{0}}, u\right) \equiv\left(T_{\sigma\left(i_{0}\right)}, v\right)$. Since $S_{i_{0}}$ and $T_{\sigma\left(i_{0}\right)}$ have the same number of vertices, and fewer than $p$ vertices, by the induction hypothesis we may write $B_{u}\left(S_{i_{0}}\right) \equiv B_{v}\left(T_{\sigma\left(i_{0}\right)}\right)$. Hence

$$
B_{u}(S)=B_{u}\left(S_{i_{0}}\right)\left\{S_{i}: i \neq i_{0}\right\} \equiv B_{v}\left(T_{\sigma\left(i_{0}\right)}\right)\left\{T_{i}: i \neq \sigma\left(i_{0}\right)\right\}=B_{v}(T) .
$$

Conversely, suppose $B_{u}(S) \equiv B_{v}(T)$. Then the length of $B_{u}(S)$ is equal to the length of $B_{v}(T)$. We will prove the conclusion by induction on this length. If each word has length 0 , then both $(S, u)$ and $(T, v)$ consist of a single vertex, hence are equivalent. Now consider length $\geq 1$. We again write

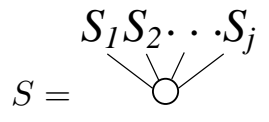

and

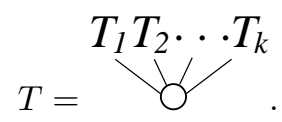

We may suppose $u \in V_{E}\left(S_{a}\right)$ and $v \in V_{E}\left(T_{b}\right)$ some $a$ and $b$. We have

$$
B_{u}\left(S_{a}\right)\left\{S_{i}: i \neq a\right\}=B_{u}(S) \equiv B_{v}(T)=B_{v}\left(T_{b}\right)\left\{T_{i}: i \neq b\right\},
$$

hence $B_{u}\left(S_{a}\right) \equiv B_{v}\left(T_{b}\right)$ and $\left\{S_{i}: i \neq a\right\} \equiv\left\{T_{i}: i \neq b\right\}$. By the induction hypothesis we have $\left(S_{a}, u\right) \equiv\left(T_{b}, v\right)$. We may therefore conclude that $(S, u) \equiv$ $(T, v)$.

The next result implies that no two distinct shuffles of a tree can appear in the same equivalence class.

Lemma 4.2. Let $(S, u),(T, v) \in \mathcal{C}$ be given. If $S \equiv T$ and $M_{u}(S) \equiv M_{v}(T)$ then $B_{u}(S) \equiv B_{v}(T)$.

Proof. By induction on $p$, where $S, T \in \mathcal{C}_{p}$. If $p=1$ then $B_{u}(S)$ and $B_{v}(T)$ are both equal to the empty word. Now consider $p>1$. Write

$$
=\underbrace{S_{1} S_{2} \ldots S_{k}}
$$

and

$$
T=\underbrace{T_{1} T_{2} \cdots T_{k}}
$$

for some $k \geq 2$. Then $u \in V_{E}\left(S_{a}\right)$ and $v \in V_{E}\left(T_{b}\right)$ for some $a$ and $b$. We wish to show $S_{a} \equiv T_{b}$. Let $x$ be the number of subtrees $T_{i}$ which are equivalent to 
$T_{b}$. Since $S$ and $T$ are equivalent, $x$ is also the number of subtrees $S_{i}$ which are equivalent to $T_{b}$. Assuming $S_{a} \not \equiv T_{b}, x$ is the number of trees which are equivalent to $T_{b}$ in $\left\{S_{i}: i \neq a\right\}$. Hence $x$ is a lower bound on the number of trees equivalent to $T_{b}$ in $M_{u}(S)=M_{u}\left(S_{a}\right) \cup\left\{S_{i}: i \neq a\right\}$. Since $M_{u}(S) \equiv M_{v}(T), x$ is a lower bound on the number of trees equivalent to $T_{b}$ in $M_{v}(T)=M_{v}\left(T_{b}\right) \cup\left\{T_{i}\right.$ : $i \neq b\}$. Since all the subtrees in $M_{v}\left(T_{b}\right)$ have fewer vertices than $T_{b}, x$ is a lower bound on the number of trees equivalent to $T_{b}$ in $\left\{T_{i}: i \neq b\right\}$. This contradicts the definition of $x$. Hence we must have $S_{a} \equiv T_{b}$ after all. Therefore $\left\{S_{i}: i \neq a\right\} \equiv\left\{T_{i}: i \neq b\right\}$, which implies $M_{u}\left(S_{a}\right) \equiv M_{v}\left(T_{b}\right)$. Since $S_{a}$ and $T_{b}$ have the same number of vertices, and fewer than $p$ vertices, we can say by the induction hypothesis that $B_{u}\left(S_{a}\right) \equiv B_{v}\left(T_{b}\right)$, and this implies

$$
B_{u}(S)=B_{u}\left(S_{a}\right)\left\{S_{i}: i \neq a\right\} \equiv B_{v}\left(T_{b}\right)\left\{T_{i}: i \neq b\right\}=B_{v}(T) .
$$

\section{Symmetry Labels and Symmetry Numbers}

Let $T$ be an element of $\mathcal{C}$, and let $v$ be a vertex of $T$. We define the symmetry label $l_{T}(v)$ of $v$ as follows: If $v$ is the root of $T$, then $l_{T}(v)=1$. If $v$ is not the root of $T$, then the height of $v$ is $k>0$ for some $k$, and there exists a unique path from the root of $T$ to $v$. Let $p_{T}(v)$ denote the vertices along this path. Let $v^{-}$be the height $k-1$ vertex in $p_{T}(v) . v^{-}$can be viewed as the "father" of $v$. Let $b_{T}(v)$ denote the set of "brothers" of $v$, namely those successors of $v^{-}$at height $k$. Let $\operatorname{sub}_{v}(T)$ be the multiset of subtrees of $T$ having a root in $b_{T}(v)$. We define $l_{T}(v)$ as the number of trees in $\operatorname{sub}_{v}(T)$ which are equivalent to the subtree having $v$ as a root. We define the symmetry labels of a marked tree $(T, v) \in(\mathcal{C}, *)$ in the same way, bearing in mind that one of the subtrees of the brothers may be marked and that no marked tree is equivalent to an unmarked tree. Figure 4.1 contains an illustration of the symmetry labels of an unmarked tree.

We define the symmetry number of a tree in $\mathcal{C} \cup(\mathcal{C}, *)$ to be the number of trees in its equivalence class. The notation is $\operatorname{sym}(T)$ for unmarked trees and $\operatorname{sym}(T, v)$ for marked trees. Symmetry labels and symmetry numbers are useful for keeping track of the multiplicities which arise when we form products of formal sums of trees.

\section{Products of Classes of Marked and Unmarked Trees}

Let $T \in \mathcal{C} \cup(\mathcal{C}, *)$. We will denote by $\operatorname{sum}(T)$ the formal sum

$$
\operatorname{sum}(T)=\sum_{T^{\prime} \equiv T} T^{\prime}
$$




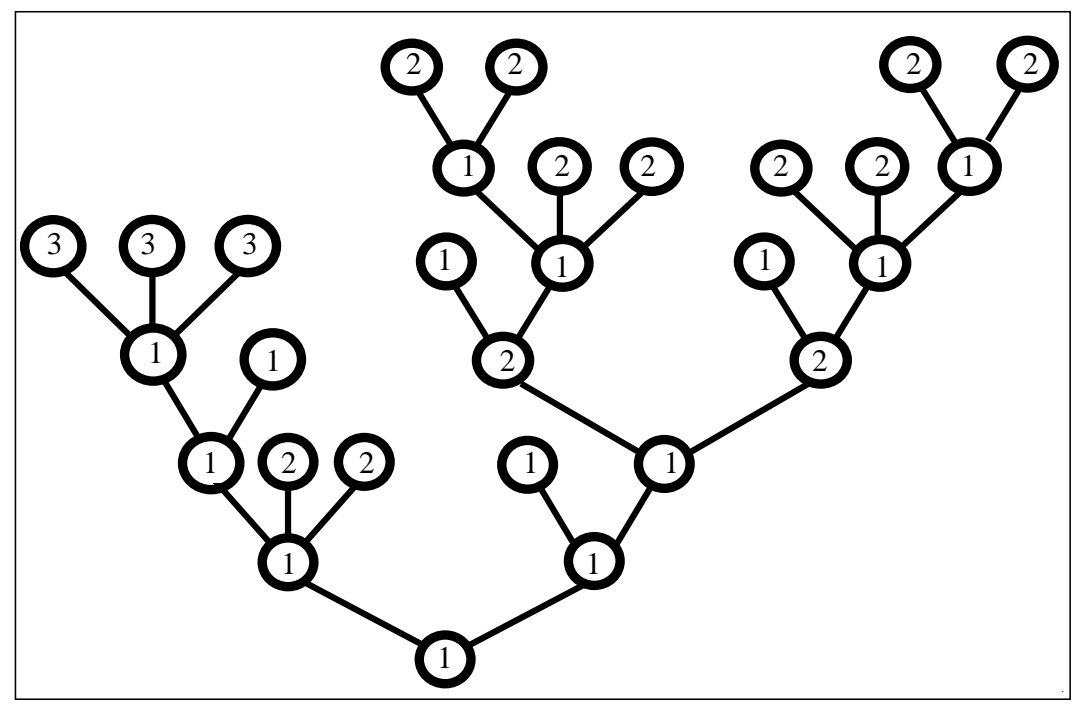

Figure 4.1: Symmetry Labels

We will multiply formal sums of trees as follows: if $(S, v) \in(\mathcal{C}, *)$ and $T \in$ $\mathcal{C} \cup(\mathcal{C}, *)$, we set

$$
\operatorname{sum}(S, v) \operatorname{sum}(T)=\sum_{\substack{\left(S^{\prime}, v^{\prime}\right) \equiv(S, v) \\ T^{\prime} \equiv T}}\left(S^{\prime}, v^{\prime}\right) T^{\prime} .
$$

We will now work out the product rules for pairs of formal sums over equivalence classes.

Lemma 4.3. Let $(R, u)$ and $(S, v)$ be marked Catalan trees, and write

$$
(R, u)(S, v)=(T, v) .
$$

Then

$$
\operatorname{sum}(R, u) \operatorname{sum}(S, v)=\operatorname{sum}(T, v) .
$$

Proof. We need to verify that every term in the product is equivalent to $(T, v)$, and that each marked tree in the class of $(T, v)$ has a unique decomposition into a product of trees, one from the class of $(R, u)$ and one from the class of $(S, v)$.

Every term in the product is equivalent to $(T, v)$ : Let $\left(R^{\prime}, u^{\prime}\right) \equiv(R, u)$ and $\left(S^{\prime}, v^{\prime}\right) \equiv(S, v)$ be given. By Lemma 4.1 , we have $B_{u^{\prime}}\left(R^{\prime}\right) \equiv B_{u}(R)$ and $B_{v^{\prime}}\left(S^{\prime}\right) \equiv B_{v}(S)$. Hence if we write $\left(R^{\prime}, u^{\prime}\right)\left(S^{\prime}, v^{\prime}\right)=\left(T^{\prime}, v^{\prime}\right)$, then

$$
B_{v^{\prime}}\left(T^{\prime}\right)=B_{v^{\prime}}\left(S^{\prime}\right) B_{u^{\prime}}\left(R^{\prime}\right) \equiv B_{v}(S) B_{u}(R)=B_{v}(T) .
$$


By Lemma 4.1 we therefore have $\left(T^{\prime}, v^{\prime}\right) \equiv(T, v)$.

Decompositions exist and are unique: Let $\left(T^{\prime}, v^{\prime}\right) \equiv(T, v)$ be given. Clearly height $\left(v^{\prime}\right)=$ height $(v)$. There is a unique path in $T^{\prime}$ from the root to $v^{\prime}$, hence a unique factorization of $\left(T^{\prime}, v^{\prime}\right)$ into $\left(R^{\prime}, u^{\prime}\right)\left(S^{\prime}, v^{\prime}\right)$ such that $u^{\prime}$ occcurs along this path and that height $\left(u^{\prime}\right)=\operatorname{height}(u)$. Since

$$
B_{v^{\prime}}\left(S^{\prime}\right) B_{u^{\prime}}\left(R^{\prime}\right)=B_{v^{\prime}}\left(T^{\prime}\right) \equiv B_{v}(T)=B_{v}(S) B_{u}(R),
$$

we must have $B_{u^{\prime}}\left(R^{\prime}\right) \equiv B_{u}(R)$ and $B_{v^{\prime}}\left(S^{\prime}\right) \equiv B_{v}(S)$. By Lemma 4.1 we must have $\left(R^{\prime}, u^{\prime}\right) \equiv(R, u)$ and $\left(S^{\prime}, v^{\prime}\right) \equiv(S, v)$.

The next lemma characterizes the product of classes of marked and unmarked trees in terms of symmetry labels.

Lemma 4.4. Let $(R, v) \in(\mathcal{C}, *)$ and $S \in \mathcal{C}$ be given, and write $(R, v) S=T$. Then

$$
\operatorname{sum}(R, v) \operatorname{sum}(S)=\left(\prod_{u \in p_{T}(v)} l_{T}(u)\right) \operatorname{sum}(T) .
$$

Proof. By induction on height $(v)$. If height $(v)=0$, then the conclusion is trivially true since the symmetry label of the root of a tree is equal to one. If height $(v)=1$, then $S$ is a height 1 subtree of $T$, and the root of $S$ as a subtree of $T$ is $v$. Write

$$
T=\underbrace{T_{1} T_{2} \cdots T_{n}}
$$

By definition of symmetry labels there are exactly $l_{T}(v)$ subtrees $T_{i}$ which are equivalent to $S$. Hence any tree $T^{\prime}$ equivalent to $T$ has exactly $l_{T}(v)$ height one subtrees which are equivalent to $S$. This implies that every $T^{\prime} \equiv T$ arises in $l_{T}(v)$ ways as a product of $\left(R^{\prime}, v^{\prime}\right) \equiv(R, v)$ and $S^{\prime} \equiv S$. Therefore

$$
\operatorname{sum}(R, v) \operatorname{sum}(S)=l_{T}(v) \operatorname{sum}(T)=\left(\prod_{u \in p_{T}(v)} l_{T}(u)\right) \operatorname{sum}(T) .
$$

Now consider height $(v)>1$. Then we can decompose $(R, v)$ into the product $\left(R^{\prime}, v^{\prime}\right)\left(R^{\prime \prime}, v\right)$, where height $\left(v^{\prime}\right)=1$. By Lemma 4.3 , we can say that

$$
\operatorname{sum}(R, v)=\operatorname{sum}\left(R^{\prime}, v^{\prime}\right) \operatorname{sum}\left(R^{\prime \prime}, v\right) .
$$

We will write $T^{\prime}=\left(R^{\prime \prime}, v\right) S$. We then have $T=\left(R^{\prime}, v^{\prime}\right) T^{\prime}$. Since the height of $v$ in $R^{\prime \prime}$ is one less than the height of $v$ in $R$, we have by the induction hypothesis that

$$
\operatorname{sum}\left(R^{\prime \prime}, v\right) \operatorname{sum}(S)=\left(\prod_{u \in p_{T^{\prime}}(v)} l_{T^{\prime}}(u)\right) \operatorname{sum}\left(T^{\prime}\right) .
$$


Hence by our height 1 result we have

$$
\begin{aligned}
\operatorname{sum}(R, v) \operatorname{sum}(S) & =\operatorname{sum}\left(R, v^{\prime}\right) \operatorname{sum}\left(R^{\prime \prime}, v\right) \operatorname{sum}(S) \\
& =\operatorname{sum}\left(R, v^{\prime}\right)\left(\prod_{u \in p_{T^{\prime}}(v)} l_{T^{\prime}}(u)\right) \operatorname{sum}\left(T^{\prime}\right) \\
& =l_{T}\left(v^{\prime}\right) \cdot\left(\prod_{u \in p_{T^{\prime}}(v)} l_{T^{\prime}}(u)\right) \operatorname{sum}(T) \\
& =\left(\prod_{u \in p_{T}(v)} l_{T}(u)\right) \operatorname{sum}(T),
\end{aligned}
$$

the last equality holding because $l_{T^{\prime}}(u)=l_{T}(u)$ for $u \in p_{T^{\prime}}(v)-\left\{v^{\prime}\right\}$ and $l_{T^{\prime}}\left(v^{\prime}\right)=1, v^{\prime}$ being the root of $T^{\prime}$.

We can use symmetry numbers to conveniently summarize the contents of the last two lemmas as follows:

Proposition 4.5. Let $(R, v) \in(\mathcal{C}, *)$ and $S \in \mathcal{C} \cup(\mathcal{C}, *)$ be given, and write $T=(R, v) S$. Then

$$
\operatorname{sum}(R, v) \operatorname{sum}(S)=\frac{\operatorname{sym}(R, v) \operatorname{sym}(S)}{\operatorname{sym}(T)} \operatorname{sum}(T) .
$$

Proof. The upshot of the last two lemmas is that

$$
\operatorname{sum}(R, v) \operatorname{sum}(S)=\alpha \cdot \operatorname{sum}(T)
$$

for some integer $\alpha$, and clearly $\alpha$ must satisfy

$$
\operatorname{sym}(R, v) \operatorname{sym}(S)=\alpha \cdot \operatorname{sym}(T) .
$$

\section{Total Ordering of Catalan Trees}

We will define a total ordering $<$ of $\mathcal{C} \cup(\mathcal{C}, *)$ as follows. We first require that $S<T$ whenever $S$ has fewer external vertices than $T$. We also require that the unmarked tree consisting of a single vertex be smaller than the marked tree having a single vertex. In general we define < recursively as follows: if $S$ and $T$ have the same number of vertices,

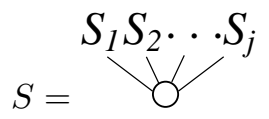

THE ELECTRonic Journal of COMBINATORICs 8 (2001), \#R2 
and

$$
T=b^{T_{1} T_{2} \ldots T_{k}},
$$

then $S<T$ if and only if the word $S_{1} S_{2} \ldots S_{j}$ is less than the word $T_{1} T_{2} \ldots T_{k}$ in lexicographic order. For example, the trees in $\mathcal{C}_{4}$ listed in increasing order are

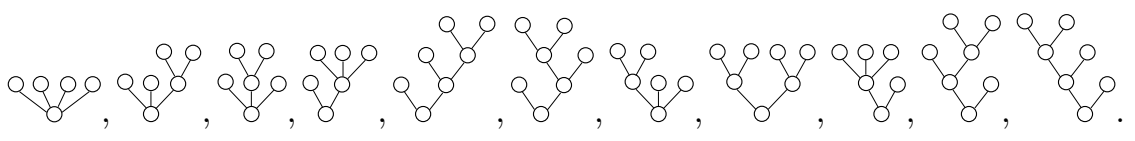

We will refer to those trees which are largest in their equivalence class as standard trees, and use them as equivalence class representatives. We will denote the set of standard Catalan trees by $\operatorname{standard}(\mathcal{C})$ and the set of standard marked Catalan trees by $\operatorname{standard}(\mathcal{C}, *)$. The standard trees in $\mathcal{C}_{4}$ are

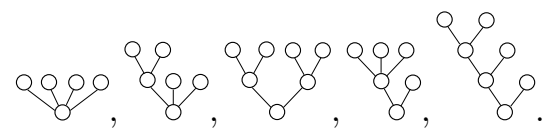

One of the standard trees in $\mathrm{CH}_{3}$ is

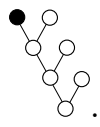

The standard tree representing the class $[T]$ is $\bar{T}$. We will also say that $[S]<[T]$ if and only if $\bar{S}<\bar{T}$.

It is not difficult to verify the following property of standard trees:

Lemma 4.6. All of the subtrees of a standard tree are standard.

\section{Chain Compositions}

We have already defined the set $C H_{k}$ of chains of height $k$. We will refine this definition by setting $\mathrm{CH}_{\left(i_{1}, \ldots, i_{k}\right)}$ equal to the the equivalence class of height $k$ chains having branch word $M_{1} M_{2} \ldots M_{k}$, where $M_{j}$ consists of $i_{j}$ trees of height zero for each $j$. For example, we have

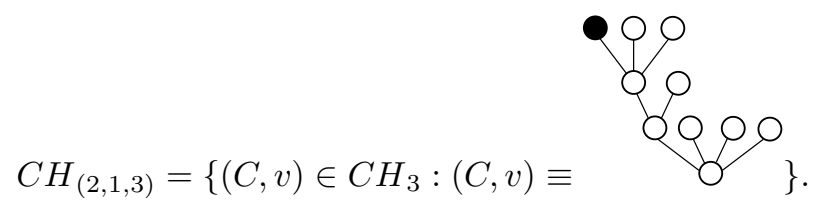


Let $M=\left\{T_{1}, \ldots, T_{r}\right\}$ be a multiset of standard Catalan trees. Then $T_{i} \equiv T_{j}$ if and only if $T_{i}=T_{j}$. Let $i_{1}, \ldots, i_{k}$ be a collection of positive integers which sum to $r$. We will denote by $C H_{\left(i_{1}, \ldots, i_{k}\right)} \circ M$ the multiset of marked trees formed in the following way: choose a chain $(C, v)$ from $C H_{\left(i_{1}, \ldots, i_{k}\right)}$, for each $i \leq r$ choose a tree $T_{i}^{\prime}$ equivalent to $T_{i}$, choose a permutation $\sigma$ of $\{1, \ldots, r\}$, and replace the $i^{\text {th }}$ unmarked external vertex of $(C, v)$ (in depth-first order) by the tree $T_{\sigma(i)}^{\prime}$. It is not difficult to see that the multiplicity of any particular tree in $\mathrm{CH}_{\left(i_{1}, \ldots, i_{k}\right)} \circ \mathrm{M}$ is $r ! / \alpha$, where $\alpha$ is the number of distinct rearrangements of the list $T_{1}, \ldots, T_{r}$, and that $(T, v) \in C H_{\left(i_{1}, \ldots, i_{k}\right)} \circ M$ implies $[(T, v)] \subset C H_{\left(i_{1}, \ldots, i_{k}\right)} \circ M$.

We will set $B_{\left(i_{1}, \ldots, i_{k}\right)}(M)$ equal to the set of branch words $M_{1} \ldots M_{k}$ which are multiset partitions of $M$ with $\left|M_{j}\right|=i_{j}$ for all $j$. Every standard $(T, v) \in$ $C H_{\left(i_{1}, \ldots, i_{k}\right)} \circ M$ satisfies $B_{v}(T) \in B_{\left(i_{1}, \ldots, i_{k}\right)}(M)$. By Lemma 4.1 we can say that if the marked trees $(S, u)$ and $(T, v)$ have distinct branch words $B_{u}(S)$ and $B_{v}(T)$, then $(S, u) \not \equiv(T, v)$. Putting this all together we have the following result:

Proposition 4.7. With notation as above,

$$
\sum_{(T, v) \in C H_{\left(i_{1}, \ldots, i_{k}\right)} \circ M}(T, v)=\frac{r !}{\alpha} \sum_{\substack{(T, v) \in \operatorname{standard}(\mathcal{C}, *) \\ B_{v}(T) \in B_{\left(i_{1}, \ldots, i_{k}\right)}(M)}} \operatorname{sum}(T, v) .
$$

\section{Linear Combinations of Formal Sums of Catalan Trees}

We can now state a theorem which is based on all the preceeding results of this section. Its purpose is to describe the multiplicities which arise when we create a formal linear combination of equivalence classes of trees by shuffling a given tree.

Let $M$ be a multiset of $r$ standard Catalan trees. Let $\alpha$ be the number of distinct rearrangements of the contents of $M$. Let $\left(i_{1}, \ldots, i_{k}\right)$ be a sequence of positive integers which sum to $r$. Let $B_{\left(i_{1}, \ldots, i_{k}\right)}(M)$ be the set of branch words $M_{1} \ldots M_{k}$ which are multiset partitions of $M$ such that $\left|M_{j}\right|=i_{j}$ for all $j$. Let $(R, v)$ be a marked Catalan tree. Let $T$ be a Catalan tree. Then

Theorem 4.8. With notation as above,

$$
\begin{gathered}
\operatorname{sum}(R, u)\left(\sum_{(S, v) \in C H_{\left(i_{1}, \ldots, i_{k}\right)} \circ M}(S, v)\right) \operatorname{sum}(T)= \\
\frac{r !}{\alpha} \underset{\substack{(S, v) \in \operatorname{standard}(\mathcal{C}, *) \\
B_{v}(S) \in B_{\left(i_{1}, \ldots, i_{k}\right)}(M)}}{\frac{\operatorname{sym}(R, u) \operatorname{sym}(S, v) \operatorname{sym}(T)}{\operatorname{sym}((R, u)(S, v) T)} \operatorname{sum}((R, u)(S, v) T) .}
\end{gathered}
$$


The multiplicity of each tree in $[(R, u)(S, v) T]$ which occurs in the right hand side of this identity is precisely

$$
\frac{r !}{\alpha} \frac{\operatorname{sym}(R, u) \operatorname{sym}(S, v) \operatorname{sym}(T)}{\operatorname{sym}((R, u)(S, v) T)} .
$$

Proof. The first statement follows from Proposition 4.7 and Proposition 4.5. To prove the second statement, let

$$
P=\left(R^{\prime}, u^{\prime}\right)\left(S^{\prime}, v^{\prime}\right) T^{\prime}
$$

and

$$
Q=\left(R^{\prime \prime}, u^{\prime \prime}\right)\left(S^{\prime \prime}, v^{\prime \prime}\right) T^{\prime \prime}
$$

be two of the terms above. We must show that

$$
P \equiv Q \Rightarrow\left(S^{\prime}, v^{\prime}\right) \equiv\left(S^{\prime \prime}, v^{\prime \prime}\right) .
$$

Assume $P \equiv Q$. Choose any vertex $w^{\prime} \in V_{E}\left(T^{\prime}\right)$. Since $T^{\prime} \equiv T^{\prime \prime}$, there must exist a corresponding vertex $w^{\prime \prime} \in V_{E}\left(T^{\prime \prime}\right)$ such that $\left(T^{\prime}, w^{\prime}\right) \equiv\left(T^{\prime \prime}, w^{\prime \prime}\right)$. This implies by Lemma 4.1 that $B_{w^{\prime}}\left(T^{\prime}\right) \equiv B_{w^{\prime \prime}}\left(T^{\prime \prime}\right)$. Since $\left(R^{\prime}, u^{\prime}\right) \equiv\left(R^{\prime \prime}, u^{\prime \prime}\right)$, we also have $B_{u^{\prime}}\left(R^{\prime}\right) \equiv B_{u^{\prime \prime}}\left(R^{\prime \prime}\right)$. Finally, we are assuming that the union of the multisets making up the branch word of $\left(S^{\prime}, v^{\prime}\right)$ is equivalent to the union of the multisets making up the branch word of $\left(S^{\prime \prime}, v^{\prime \prime}\right)$, i.e. that they are both equivalent to $M$. Hence

$$
\begin{gathered}
M_{w^{\prime}}(P)=M_{w^{\prime}}\left(T^{\prime}\right) \cup M_{v^{\prime}}\left(S^{\prime}\right) \cup M_{u^{\prime}}\left(R^{\prime}\right) \equiv \\
M_{w^{\prime \prime}}\left(T^{\prime \prime}\right) \cup M_{v^{\prime \prime}}\left(S^{\prime \prime}\right) \cup M_{u^{\prime \prime}}\left(R^{\prime \prime}\right)=M_{w^{\prime \prime}}(Q) .
\end{gathered}
$$

By Lemma 4.2 we must conclude that

$$
B_{w^{\prime}}(P) \equiv B_{w^{\prime \prime}}(Q) .
$$

Therefore

$$
B_{w^{\prime}}\left(T^{\prime}\right) B_{v^{\prime}}\left(S^{\prime}\right) B_{u^{\prime}}\left(R^{\prime}\right) \equiv B_{w^{\prime \prime}}\left(T^{\prime \prime}\right) B_{v^{\prime \prime}}\left(S^{\prime \prime}\right) B_{u^{\prime \prime}}\left(R^{\prime \prime}\right) .
$$

This forces

$$
B_{v^{\prime}}\left(S^{\prime}\right) \equiv B_{v^{\prime \prime}}\left(S^{\prime \prime}\right)
$$

By Lemma 4.1 again we therefore have $\left(S^{\prime}, v^{\prime}\right) \equiv\left(S^{\prime \prime}, v^{\prime \prime}\right)$.

As an immediate application of this theorem we can carry out the computations in Section 3 in a more general setting. Let

$$
C_{a, b}^{(k)}\left(x_{1}, \ldots, x_{n}\right)=\sum_{X \in C H_{k}} w_{a, b}(X)=(a, b) \text {-entry of }(-1)^{k}\left(\frac{\partial H_{i}}{\partial x_{j}}\right)^{k}
$$


and

$$
A_{i}\left(x_{1}, \ldots, x_{n}, q_{1}, \ldots, q_{r}\right)=\sum_{T_{j} \in M} w_{i}\left[T_{j}\right] q_{j}
$$

where the $q_{j}$ are indeterminants.

Theorem 4.9. With notation as above, the coefficient of $q_{1} q_{2} \cdots q_{r}$ in the expression

$$
\sum_{1 \leq a, b \leq n} w_{i, a}[R, v] C_{a, b}^{(k)}\left(A_{1}, \ldots, A_{n}\right) w_{b}[T]
$$

is

$$
\begin{gathered}
\frac{r !}{\alpha} \sum_{\substack{i_{1}+\cdots+i_{k}=r \\
(S, v) \in \operatorname{standard}(\mathcal{C}, *)}} \frac{\operatorname{sym}(R, u) \operatorname{sym}(S, v) \operatorname{sym}(T)}{\operatorname{sym}((R, u)(S, v) T)} w_{i}[(R, u)(S, v) T] . \\
B_{v}(S) \in B_{\left(i_{1}, \ldots, i_{k}\right)}(M)
\end{gathered}
$$

Corollary 4.10. With notation as above, if $H_{i}$ is homogeneous of total degree $d+1$ for each $i$ and $(\partial H)^{k}=0$ then

$$
\sum_{\substack{(S, v) \in \operatorname{standard}(\mathcal{C}, *) \\ B_{v}(S) \in B_{d^{k}}(M)}} \frac{\operatorname{sym}(S, v)}{\operatorname{sym}((R, u)(S, v) T)} w_{i}[(R, u)(S, v) T]=0 .
$$

In order to perform Gaussian elimination on such expressions, we need to identify the smallest term of the form $[(R, u)(S, v) T]$. This is the goal of the next section.

\section{The Smallest Shuffle of a Tree}

We will define two partial orders on multisets of standard trees as follows: $M_{1} \leq$ $M_{2}$ if and only if there is an injection $\phi: M_{1} \rightarrow M_{2}$ such that $T \leq \phi(T)$ for all $T \in M_{1}$, and $M_{1} \preceq M_{2}$ if and only if $S \leq T$ for all $S \in M_{1}$ and $T \in M_{2}$. Given a multiset $M$ of $r$ standard trees, and given a partition $\left(i_{1}, \ldots, i_{k}\right)$ of $r$, there is a unique multiset partition $M_{1}, \ldots, M_{k}$ of $M$ such that $\left|M_{j}\right|=i_{j}$ for all $j$ and $M_{1} \preceq \cdots \preceq M_{k}$ : place the $i_{1}$ smallest trees of $M$ in $M_{1}$, place the next $i_{2}$ smallest trees in $M_{2}$, and so on. Our goal in this section is to prove Theorem 4.11 below. Recall our notation that $\bar{T}$ is the largest tree in the class of $T$. We will also represent the tree

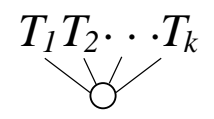

by the vector $\left(T_{1}, T_{2}, \ldots, T_{k}\right)$. 
Theorem 4.11. Let $i_{1}, \ldots, i_{k}$ be a collection of positive integers. Let $M$ be a multiset of standard trees of cardinality $i_{1}+\cdots+i_{k}$. Let $(S, v)$ be any marked tree with $B_{v}(S)=M_{1} \ldots M_{k} \in B_{\left(i_{1}, \ldots, i_{k}\right)}(M)$, where $M_{1} \preceq \ldots \preceq M_{k}$. Let $(R, u)$ and $T$ be given trees. Then

$$
\overline{(R, u)(S, v) T}
$$

is the smallest tree in

$$
\left\{\overline{(R, u)\left(S^{\prime}, v^{\prime}\right) T}: B_{v^{\prime}}\left(S^{\prime}\right) \in B_{\left(i_{1}, \ldots, i_{k}\right)}(M)\right\} .
$$

In order to prove this theorem we will need the following lemmas.

Lemma 4.12. Let $X$ be a totally ordered set. For each word $w$ in $X^{*}$, let $\bar{w}$ denote the largest rearrangement of $w$ in lexicographic order. If $w$ and $w^{\prime}$ are two words in $X^{*}$ of the same length, $x \in X$, and $\bar{w} \geq \overline{w^{\prime}}$, then $\overline{w x} \geq \overline{w^{\prime} x}$.

Proof. By induction on length $(w)$. The case length $(w)=0$ is trivially true. Consider length $(w) \geq 1$. Write $\bar{w}=a_{1} \cdots a_{k}$ and $\overline{w^{\prime}}=b_{1} \cdots b_{k}$. Then $a_{1} \geq$ $\cdots a_{k}$ and $b_{1} \geq \cdots \geq b_{k}$. There are three cases to consider. If $x \geq a_{1} \geq b_{1}$ then $\overline{w x}=x a_{1} \cdots a_{k} \geq x b_{1} \cdots b_{k}=\overline{w^{\prime} x}$. If $a_{1}>x \geq b_{1}$ then $\overline{w x} \geq a_{1} \cdots a_{k} x>$ $x b_{1} \cdots b_{k}=\overline{w^{\prime} x}$. If $a_{1} \geq b_{1}>x$ then write $\bar{w}=a_{1} W$ and $\overline{w^{\prime}}=b_{1} W^{\prime}$. Then $\overline{w x}=a_{1} \overline{W x}$ and $\overline{w^{\prime} x}=b_{1} \overline{W^{\prime} x}$. If $a_{1}>b_{1}$ then clearly $\overline{w x}>\overline{w^{\prime} x}$. On the other hand, if $a_{1}=b_{1}$ then $W \geq W^{\prime}$, hence by the induction hypothesis $\overline{W x} \geq \overline{W^{\prime} x}$, and this implies $\overline{w x} \geq \overline{w^{\prime} x}$.

Corollary 4.13. Let $M$ and $N$ be multisets of standard trees of equal cardinality $\geq 2$. Assume the standard tree whose height one subtrees make up $M$ is greater than or equal to the standard tree whose height one subtrees make up $N$. Let $T$ be a standard tree. Then the standard tree whose height one subtrees make up $M \cup\{T\}$ is greater than or equal to the standard tree whose height one subtrees make up $N \cup\{T\}$.

Proof. In general, if the standard trees in a multiset $X$ are $T_{1} \geq T_{2} \geq \cdots \geq T_{k}$, then the standard tree having these height one subtrees is $\left(T_{1}, T_{2}, \cdots, T_{k}\right)$, which is the largest rearrangement of the word $T_{1} T_{2} \cdots T_{k}$ in lexicographic order.

Lemma 4.14. Let $M$ and $N$ be multisets of standard trees of equal cardinality $\geq 2$ such that $M \leq N$. Let $S$ be the standard tree whose height one subtrees make up the multiset $M$, and let $T$ be the standard tree whose height one subtrees make up the multiset $N$. Then $S \leq T$. 
Proof. Since $M \leq N$, there is an injection $\phi: M \rightarrow N$ such that $S \leq \phi(S)$ for all $S \in M$. Let $S_{1} \geq S_{2} \geq \cdots \geq S_{k}$ be the trees in $M$, and let $T_{1} \geq T_{2} \geq \cdots \geq T_{k}$ be the trees in $N$. As words in $\operatorname{standard}(\mathcal{C})^{*}$ we have

$$
S_{1} S_{2} \cdots S_{k} \leq \phi\left(S_{1}\right) \phi\left(S_{2}\right) \cdots \phi\left(S_{k}\right) \leq T_{1} T_{2} \cdots T_{n},
$$

hence

$$
S=\left(S_{1}, S_{2}, \ldots, S_{k}\right) \leq\left(T_{1}, T_{2}, \ldots, T_{k}\right)=T .
$$

Lemma 4.15. Let $(R, u)$ and $(S, v)$ be marked trees whose branch multisets $M_{u}(R)$ and $M_{v}(S)$ consist of standard trees, assume

$$
\operatorname{height}(u)=\operatorname{height}(v)=p,
$$

write $B_{u}(R)=M_{1} M_{2} \ldots M_{p}$ and $B_{v}(S)=N_{1} N_{2} \ldots N_{p}$, and assume $M_{i} \leq N_{i}$ for all $i$. Then for all $T_{1} \leq T_{2} \in \operatorname{standard}(\mathcal{C})$ we have

$$
\overline{(R, u) T_{1}} \leq \overline{(S, v) T_{2}} .
$$

Proof. By induction on $p$. If $p=1$ then we appeal to Lemma 4.14. If $p>1$, write

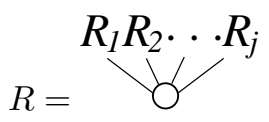

and

$$
S=\underbrace{S_{1} S_{2} \ldots S_{k}} .
$$

Let $R_{a}$ be the subtree which contains $u$. Then $B_{u}\left(R_{a}\right)=M_{1} \ldots M_{p-1}$ and $M_{p}=\left\{R_{i}: i \neq a\right\}$. Let $S_{b}$ be the subtree which contains $v$. Then $B_{v}\left(S_{b}\right)=$ $N_{1} \ldots N_{p-1}$ and $N_{p}=\left\{S_{i}: i \neq b\right\}$. By the induction hypothesis, we have $\overline{\left(R_{a}, u\right) T_{1}} \leq \overline{\left(S_{b}, v\right) T_{2}}$. Since $M_{p} \leq N_{p}$, there is an injection

$$
\phi:\left\{R_{i}: i \neq a\right\} \rightarrow\left\{S_{i}: i \neq b\right\}
$$

which satisfies $x \leq \phi(x)$ for all $x \in M_{p}$. We can extend $\phi$ to an injection from $\left\{R_{i}: i \neq a\right\} \cup\left\{\overline{\left(R_{a}, u\right) T_{1}}\right\}$ to $\left\{S_{i}: i \neq b\right\} \cup\left\{\overline{\left(S_{b}, v\right) T_{2}}\right\}$ by setting

$$
\phi\left(\overline{\left(R_{a}, u\right) T_{1}}\right)=\overline{\left(S_{b}, v\right) T_{2}} .
$$

This is an injection from the height one subtrees of $\overline{(R, u) T_{1}}$ to the height one subtrees of $\overline{(S, v) T_{2}}$. Hence by Lemma 4.14 we can say $\overline{(R, u) T_{1}} \leq \overline{(S, v) T_{2}}$. 
Proof of Theorem 4.11. There is no harm in assuming that $(R, u)$ and $T$ are standard, because the result we are proving is a statement about equivalence classes. Having made this assumption, by Lemma 4.15 if will suffice to show that

$$
\overline{(S, v) T}
$$

is the smallest tree in

$$
\left\{\overline{\left(S^{\prime}, v^{\prime}\right) T}: B_{v^{\prime}}\left(S^{\prime}\right) \in B_{\left(i_{1}, \ldots, i_{k}\right)}(M)\right\} .
$$

We will prove this by induction on $q=i_{1}+\cdots+i_{k}$.

If $k=1$ then the only tree in

$$
\left\{\overline{\left(S^{\prime}, v^{\prime}\right) T}:\left(S^{\prime}, v^{\prime}\right) \in B_{\left(i_{1}, \ldots, i_{k}\right)}(M)\right\}
$$

is $\overline{\left(T, T_{1}, \ldots, T_{i_{1}}\right)}$, where $M=\left\{T_{1}, \ldots, T_{i_{1}}\right\}$, and the conclusion follows. This includes the base case $q=1$.

We now assume $k \geq 2$. We will say that $Z$ is the largest tree in $M$. By definition of $(S, v), B_{v}(S)=M_{1} M_{2} \cdots M_{k} \in B_{\left(i_{1}, \ldots, i_{k}\right)}(M)$, where $M_{1} \preceq M_{2} \preceq$ $\cdots \preceq M_{k}$. Hence $Z \in M_{k}$. Let $\left(S^{\prime}, v^{\prime}\right) \in(\mathcal{C}, *), B_{v^{\prime}}\left(S^{\prime}\right) \in B_{\left(i_{1}, \ldots, i_{k}\right)}(M)$ be given. Write $B_{v^{\prime}}\left(S^{\prime}\right)=N_{1} N_{2} \cdots N_{k}$. Let $S_{1}$ be the height 1 subtree of $S$ which contains the vertex $v$. Let $S_{1}^{\prime}$ be the height 1 subtree of $S^{\prime}$ which contains the vertex $v^{\prime}$. Then we have $B_{v}\left(S_{1}\right)=M_{1} \cdots M_{k-1}$ and $B_{v^{\prime}}\left(S_{1}^{\prime}\right)=N_{1} \cdots N_{k-1}$. There are two cases to consider, $Z \in N_{k}$ and $Z \notin N_{k}$.

Case 1. Suppose $Z \in N_{k}$. If $i_{k}=1$, then $\bigcup_{j=1}^{k-1} M_{j}=\bigcup_{j=1}^{k-1} N_{j}$, hence by the induction hypothesis $\overline{\left(S_{1}^{\prime}, v^{\prime}\right) T} \geq \overline{\left(S_{1}, v\right) T}$. Since $\overline{\left(S^{\prime}, v^{\prime}\right) T}$ has height one subtrees consisting of $\overline{\left(S_{1}^{\prime}, v^{\prime}\right) T}$ and $Z$, and $\overline{(S, v) T}$ has height one subtrees consisting of $\overline{\left(S_{1}, v\right) T}$ and $Z$, we have by Lemma 4.14 that $\overline{\left(S^{\prime}, v^{\prime}\right) T} \geq \overline{(S, v) T}$.

Now consider $i_{k}>1$. We will apply the induction hypothesis to the situation in which $i_{k}$ is replaced by $i_{k}-1$. Let $M_{k}^{\prime}$ and $N_{k}^{\prime}$ be the multisets obtained from $M_{k}$ and $N_{k}$ respectively by removing one copy of $Z$ from each. Let $P$ be the standard tree whose height one subtrees make up the multiset $M_{k}^{\prime} \cup\left\{\overline{\left(S_{1}, v\right) T}\right\}$ and let $Q$ be the standard tree whose height one subtrees make up the multiset $N_{k}^{\prime} \cup\left\{\overline{\left(S_{1}^{\prime}, v^{\prime}\right) T}\right\}$. By the induction hypothesis, $Q \geq P$. Since the height one subtrees of $\overline{(S, v) T}$ make up $M_{k} \cup\left\{\overline{\left(S_{1}, v\right) T}\right\}$, and the height one subtrees of $\overline{\left(S^{\prime}, v^{\prime}\right) T}$ make up $N_{k} \cup\left\{\overline{\left(S_{1}^{\prime}, v^{\prime}\right) T}\right\}$, we have by Corollary 4.13 (with the tree $Z$ playing the role of the inserted tree) that $\overline{\left(S^{\prime}, v^{\prime}\right) T} \geq \overline{(S, v) T}$.

Case 2. Next consider $Z \notin N_{k}$. Then $Z$ lives somewhere in $S_{1}^{\prime}$, and this will lead us to our conclusion after we make a reduction which brings us back to Case 1. There is some $Y<Z$ which lies in $N_{k}$, and there is a copy of $Z$ in some $N_{i}, i<k$. Let $\left(S^{\prime \prime}, v^{\prime}\right)$ be the tree obtained from $\left(S^{\prime}, v^{\prime}\right)$ obtained by exchanging the positions of these copies of $Z$ and $Y$ as they occur on the chain $C_{v^{\prime}}\left(S^{\prime}\right)$ of $\left(S^{\prime}, v^{\prime}\right)$. Let $S_{1}^{\prime \prime}$ be the height 1 one subtree of $S^{\prime \prime}$ which contains $v^{\prime}$. We will show that $\overline{\left(S^{\prime}, v^{\prime}\right) T} \geq \overline{\left(S^{\prime \prime}, v^{\prime}\right) T}$ and $\overline{\left(S^{\prime \prime}, v^{\prime}\right) T} \geq \overline{(S, v) T}$. 
The tree $\left(S_{1}^{\prime \prime}, v^{\prime}\right)$ can be obtained from $\left(S_{1}^{\prime}, v^{\prime}\right)$ by replacing one copy of $Z$ with $Y$ along $C_{v^{\prime}}\left(S_{1}^{\prime \prime}\right)$, and by Lemma 4.15 , we can say that $\overline{\left(S_{1}^{\prime}, v^{\prime}\right) T} \geq$ $\overline{\left(S_{1}^{\prime \prime}, v^{\prime}\right) T}$. Moreover, since $\overline{\left(S_{1}^{\prime}, v^{\prime}\right) T}$ contains $Z$ as a proper subtree and $Z$ is the largest tree in $M, \overline{\left(S_{1}^{\prime}, v^{\prime}\right) T}$ is larger than any tree in $M$. Hence $\overline{\left(S_{1}^{\prime}, v^{\prime}\right) T}$ is a height one subtree of $\overline{\left(S^{\prime}, v^{\prime}\right) T}$ which is at least as large (in fact it is strictly larger) than any height one subtree of $\overline{\left(S^{\prime \prime}, v^{\prime}\right) T}$. Hence $\overline{\left(S^{\prime}, v^{\prime}\right) T} \geq \overline{\left(S^{\prime \prime}, v^{\prime}\right) T}$, because the word consisting of the height 1 subtrees of $\overline{\left(S^{\prime}, v^{\prime}\right) T}$ is at least as large as the word consisting of the height 1 subtrees of $\overline{\left(S^{\prime \prime}, v^{\prime}\right) T}$ in lexicographic order. If we write $B_{v^{\prime}}\left(S^{\prime \prime}\right)=N_{1}^{\prime} N_{2}^{\prime} \cdots N_{k}^{\prime}$, then we have $Z \in N_{k}^{\prime}$ by construction. By Case 1 considered above, we have $\overline{\left(S^{\prime \prime}, v^{\prime}\right) T} \geq \overline{(S, v) T}$. Hence $\overline{\left(S^{\prime}, v^{\prime}\right) T} \geq$ $\overline{(S, v) T}$. This completes the proof.

Let $X$ be a finite set of standard Catalan trees. Let $L$ be a linear combination of the form

$$
L=\sum_{T \in X} \alpha(T) \operatorname{sum}(T),
$$

where each $\alpha(T)$ is a non-zero scalar. We will say that the leading term of $L$ is the smallest tree in $X$. We can combine Theorem 4.8 with Theorem 4.11 to obtain the following result:

Theorem 4.16. Let $M$ be a multiset of standard Catalan trees of cardinality $r$, let $\left(i_{1}, \ldots, i_{k}\right)$ be a partition of $r$, let $(R, u)$ be a marked Catalan tree and $T$ an unmarked Catalan tree. Then

$$
\operatorname{sum}(R, u)\left(\sum_{(S, v) \in C H_{\left(i_{1}, \ldots, i_{k}\right)} \circ M}(S, v)\right) \operatorname{sum}(T)
$$

is a linear combination over equivalence classes of Catalan trees with leading term

$$
\overline{(R, u)(S, v) T},
$$

where $(S, v)$ is that unique standard tree which satisfies

$$
B_{v}(S)=M_{1} \cdots M_{k} \in B_{\left(i_{1}, \ldots, i_{k}\right)}(M)
$$

and $M_{1} \preceq \cdots \preceq M_{k}$.

Having established the basic vocabulary and combinatorial properties of Catalan trees, we move on to identify those properties of Catalan trees which enable us to prove Theorem 1.1.

\section{Applications to the Jacobian Conjecture}

We will refer to any unmarked standard tree $T$ for which there exists a vertex $v \in V_{E}(T)$ such that $B_{v}(T)=M_{1} \cdots M_{j}$ and $M_{a} \preceq \cdots \preceq M_{a+k-1}$ for some $a$ 
as being $k$-good. Any standard tree which is not $k$-good is termed $k$-bad. If $(T, v)$ is a marked tree such that $M_{v}(T)$ consists of standard trees and $B_{v}(T)=$ $M_{1} \cdots M_{k}$ and $M_{1} \preceq \cdots \preceq M_{k}$, we will refer to $(T, v)$ as being especially $k$ good. Every $k$-good tree $T$ can be factored as $T=(Q, u)(R, v) S$, where $(R, v)$ is especially $k$-good. Theorem 4.16 tells us that given this decomposition of $T$, the leading term of

$$
\operatorname{sum}(Q, u)\left(\sum_{\left(R^{\prime}, v^{\prime}\right) \in C H_{\left(i_{1}, \ldots, i_{k}\right)} \circ M}\left(R^{\prime}, v^{\prime}\right)\right) \operatorname{sum}(S)
$$

is $T$, where $M=M_{v}(R), B_{v}(R)=M_{1} \cdots M_{k}$, and $\left|M_{j}\right|=i_{j}$ for all $j$.

Every standard tree $T$ with height $\geq 2$ is 2 -good: let $v \in V_{E}(T)$ be such that height $(v)=\operatorname{height}(T)$. Write $B_{v}(T)=M_{1} M_{2} \cdots M_{k}$. Then $M_{1}$ consists of height 0 trees, hence $M_{1} \preceq M_{2}$. Hence every standard tree $T$ of height $\geq 2$ occurs as the leading term of some linear combination of the type found in equation 5.1. Hence by Gaussian elimination we have

Theorem 5.1. The set of linear combinations of the form

$$
\operatorname{sum}(Q, u)\left(\sum_{\substack{(R, v) \in C H_{\left(i_{1}, i_{2}\right)} \circ M \\(Q, u)(R, v) S \in \mathcal{C}_{p}}}(R, v)\right) \operatorname{sum}(S)
$$

spans the set

$$
\left\{\operatorname{sum}(T): T \in \mathcal{C}_{p} \& \operatorname{height}(T) \geq 2\right\}
$$

Note that if the polynomial system $H=\left(H_{1}, H_{2}, \ldots, H_{n}\right)$ is homogeneous of total degree $d$, then $w_{i}(T)=0$ for any tree $T$ which has an internal vertex with out-degree other than $d$. Moreover, the weight of the tree

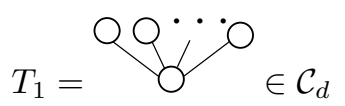

is

$$
w_{i}\left(T_{1}\right)=-H_{i},
$$

the weight of the tree $T_{2}=\left(T_{1}, T_{1}, \ldots, T_{1}\right)$ is

$$
w_{i}\left(T_{2}\right)=(-1)^{d+1} H_{i} \circ H,
$$

the weight of the tree $T_{3}=\left(T_{2}, T_{2}, \ldots, T_{2}\right)$ is

$$
w_{3}\left(T_{3}\right)=(-1)^{d^{2}+d+1} H_{i} \circ H \circ H,
$$

and so on. If we combine Theorem 5.1 with Theorem 4.8 and Corollary 4.10, we obtain 
Theorem 5.2. If $H_{i}$ is homogeneous of degree $d$ for each $i$ and $\left(\frac{\partial H_{i}}{\partial x_{j}}\right)^{2}=0$, then $w_{i}[T]=0$ for all $T \in \mathcal{C}$ such that height $(T) \geq 2$. Hence the inverse of $F=\left(x_{1}+H_{1}, x_{2}+H_{2}, \ldots, x_{n}+H_{n}\right)$ is $G=\left(x_{1}-H_{1}, x_{2}-H_{2}, \ldots, x_{n}-H_{n}\right)$ and $H \circ H=0$.

We will define the set $\mathcal{B}$ of binary Catalan trees as those Catalan trees all of whose internal vertices have out-degree equal to two. Our remaining task is to prove the following result about binary Catalan trees:

Theorem 5.3. The set of linear combinations of the form

$$
\operatorname{sum}(Q, u)\left(\sum_{\substack{(R, v) \in C H_{(1,1,1)} \circ M \\(Q, u)(R, v) S \in \mathcal{B}_{p}}}(R, v)\right) \operatorname{sum}(S)
$$

spans the set

$$
\left\{\operatorname{sum}(T): T \in \mathcal{B}_{p}\right\}
$$

whenever $p \geq 7$.

If we combine Theorem 5.3 with Theorem 4.8 and Corollary 4.10, we obtain

Theorem 5.4. If $H_{i}$ is homogeneous of total degree 2 for each $i$ and $\left(\frac{\partial H_{i}}{\partial x_{j}}\right)^{3}=0$ then $w_{i}[T]=0$ for all $T \in \bigcup_{p=7}^{\infty} \mathcal{B}_{p}$. Hence the inverse of $F=\left(x_{1}+H_{1}, x_{2}+\right.$ $\left.H_{2}, \ldots, x_{n}+H_{n}\right)$ is a polynomial system of degree $\leq 6$ and $H \circ H \circ H=0$.

In order to prove Theorem 5.3, we will first list a few easily verified facts about standard binary Catalan trees. We will omit the proof of Lemmas 5.5 through 5.7.

Lemma 5.5. The binary tree

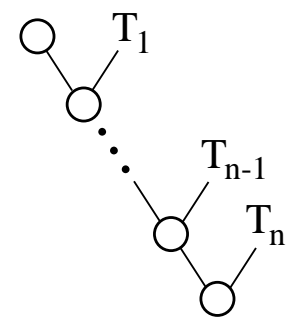


is standard if and only if $T_{1}=\mathrm{O}$, each $T_{i}$ is standard, and

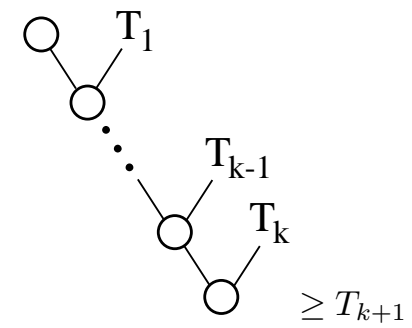

for all $1 \leq k<n$.

Lemma 5.6. If the binary tree

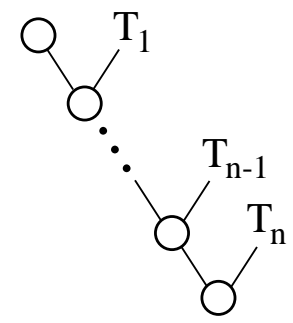

is standard and has greater than $2^{k}$ external vertices, then $n>k$.

Lemma 5.7. Every subtree of a $k$-bad tree is $k$-bad.

Lemma 5.8. Let $T$ be a 3 -bad standard binary tree with $\geq 7$ external vertices. Then

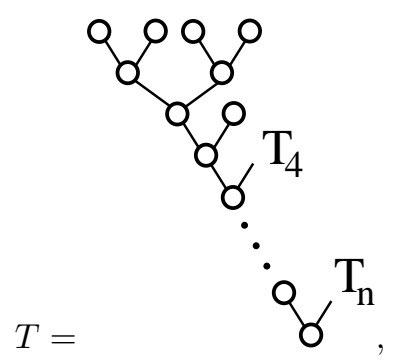

where

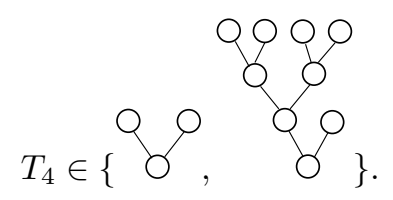


Proof. By Lemma 5.6, we know that

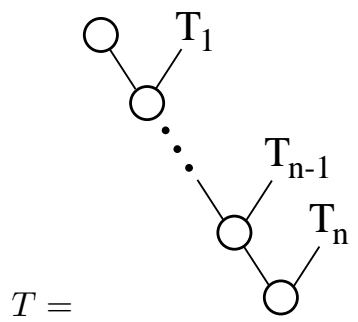

for some $n \geq 3$. By Lemma $5.5, T_{1}=$ o. Since $T$ is 3 -bad, $T_{2}$ cannot be equal to o. By Lemma 5.5,

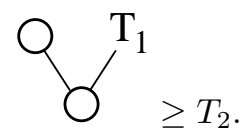

Hence

$$
T_{2}=\text { qo }
$$

Since $T$ is 3 -bad, the only choice for $T_{3}$ is $T_{3}=$ o. Since $T$ has at least seven vertices, $n \geq 4$. We now have

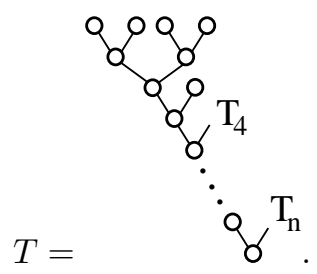

By Lemma 5.5,

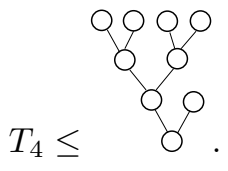

That leaves us with

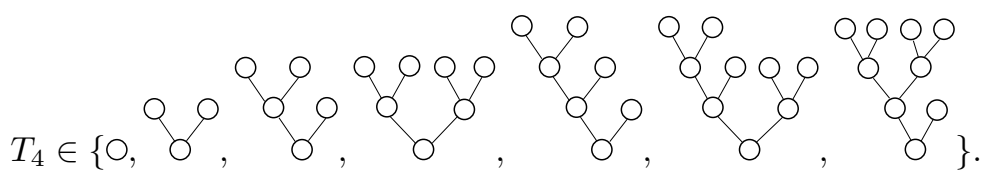

By Lemma 5.7, $T_{4}$ is 3-bad Hence

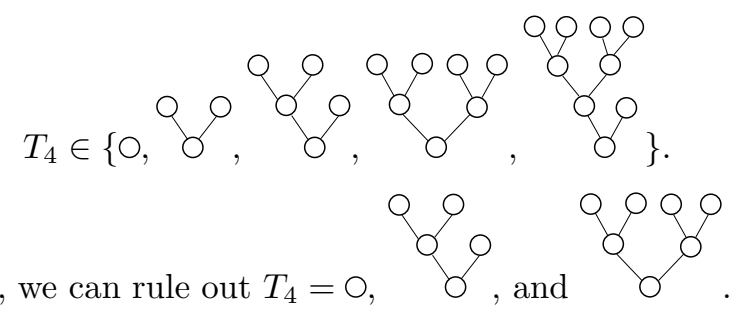

Since $T$ is 3 -bad, we can rule out $T_{4}=0$,

THE ElECTRONIC JOURNAL OF COMBINATORICS 8 (2001), \#R2 
Proof of Theorem 5.3. Every standard 3-good binary tree $T$ has a factorization of the form $T=(Q, u)(R, v) S$, where $(R, v)$ is especially 3-good. Therefore $T$ occurs as the leading term in a linear combination of the type found in 5.2. We will call such linear combinations 3-good combinations. Hence we only need to show that every standard 3-bad binary tree having $\geq 7$ vertices occurs as the leading term of some linear combination of 3-good combinations. By Gaussian elimination this will imply that every expression of the form $\operatorname{sum}(T)$, $T \in \bigcup_{p=7}^{\infty} \mathcal{B}_{p}$, lies in the span of all 3-good combinations.

By Lemma 5.8, every 3-bad standard binary tree having $\geq 7$ vertices factors as either $(R, v) T_{1}$ or $(R, v) T_{2}$ for some marked tree $(R, v)$, where

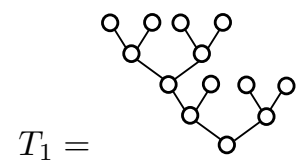

and

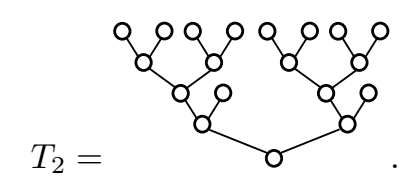

If we can show that $T_{1}$ is the leading term of a linear combination of 3 -good combinations of the form

$$
\sum_{i} \alpha_{i} L_{i}
$$

and that $T_{2}$ is the leading term of a linear combination of 3-good combinations of the form

$$
\sum_{i} \beta_{i} L_{i}
$$

then every 3 -bad standard binary tree with $\geq 7$ vertices will appear as the leading term in an expression of the form

$$
\sum_{i} \alpha_{i} \cdot \operatorname{sum}(R, v) L_{i}
$$

or

$$
\sum_{i} \beta_{i} \cdot \operatorname{sum}(R, v) L_{i},
$$

both of which are linear combinations of 3-good combinations. Note that we are using Lemma 4.15 to say that if the leading term of $\sum_{i} \gamma_{i} L_{i}$ is $T$, then the leading term of $\sum_{i} \gamma_{i} \cdot \operatorname{sum}(R, v) L_{i}$ is $\overline{(R, v) T}$.

The next smallest standard binary tree before $T_{1}$ is

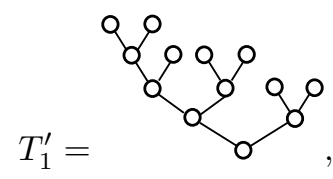

THE EleCtronic Journal of COMBinatorics 8 (2001), \#R2 
which is 3 -good. $T_{1}^{\prime}$ can be factored in two ways:

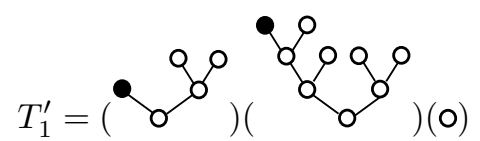

and

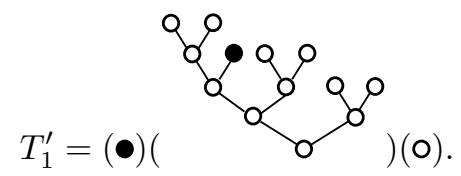

Hence $T_{1}^{\prime}$ is the leading term of both

$$
L_{1}=\operatorname{sum}\left(\cos ^{\rho}\right)\left(\sum_{(R, v) \in C H_{(1,1,1)} \circ\left\{0,0, \rho_{\}}\right\}}^{(R, v)}\right) \operatorname{sum}(0)
$$

and

$$
L_{2}=\operatorname{sum}(\bullet)\left(\sum_{(R, v) \in C H_{(1,1,1)} \circ\{\delta, \delta, \delta\}}(R, v)\right) \operatorname{sum}(0) .
$$

By Theorem 4.8, we can compute

$$
L_{1}=\frac{3 !}{3}\left(4 \cdot \operatorname{sum}\left(T_{1}^{\prime}\right)+2 \cdot \operatorname{sum}\left(T_{1}\right)+\operatorname{sum}(\overbrace{0})\right)
$$

and

$$
L_{2}=\frac{3 !}{1} \operatorname{sum}\left(T_{1}^{\prime}\right)
$$

Hence

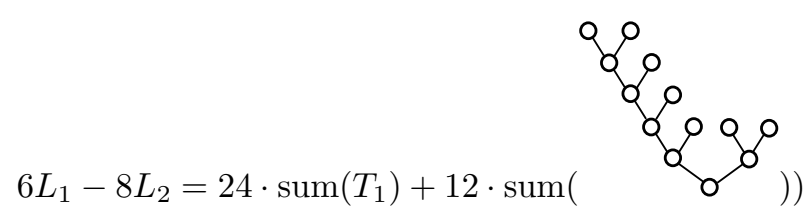

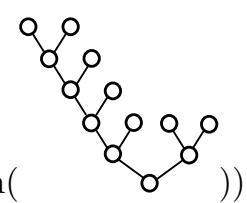

is a linear combination with leading term $T_{1}$ and is in the span of all 3-good combinations.

The next smallest standard binary Catalan tree before $T_{2}$ is

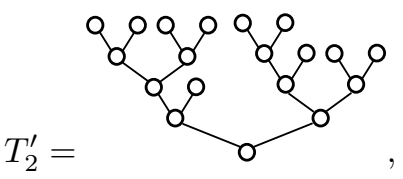

THE ElECTRONic JOURNAL OF COMBINATORICS 8 (2001), \#R2 
which is 3 -good. $T_{2}^{\prime}$ can be factored in two ways:

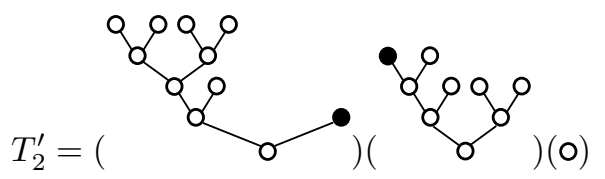

and

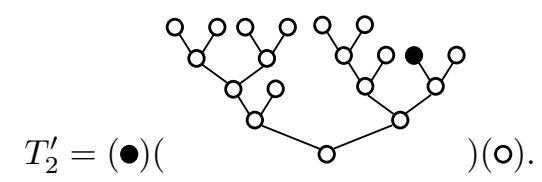

Hence $T_{2}^{\prime}$ is the leading term of both

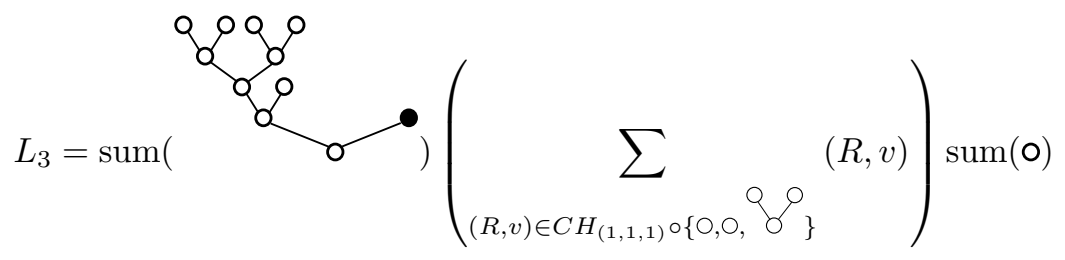

and

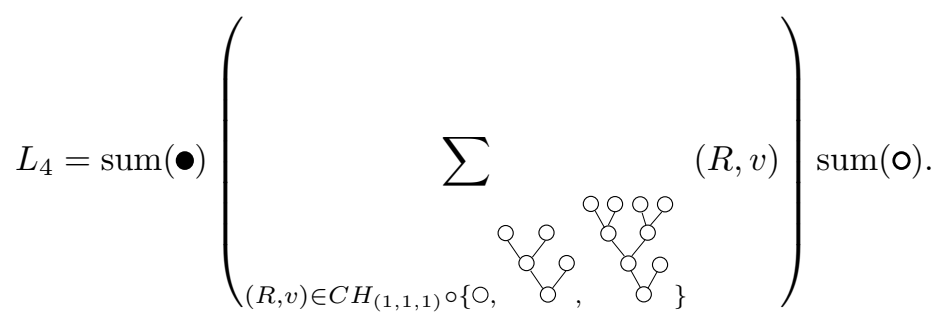

By Theorem 4.8, we can compute

$$
L_{3}=\frac{3 !}{3}\left(2 \cdot \operatorname{sum}\left(T_{2}^{\prime}\right)+4 \cdot \operatorname{sum}\left(T_{2}\right)+\operatorname{sum}(\underbrace{\text { go gog }}_{0})\right)
$$

and

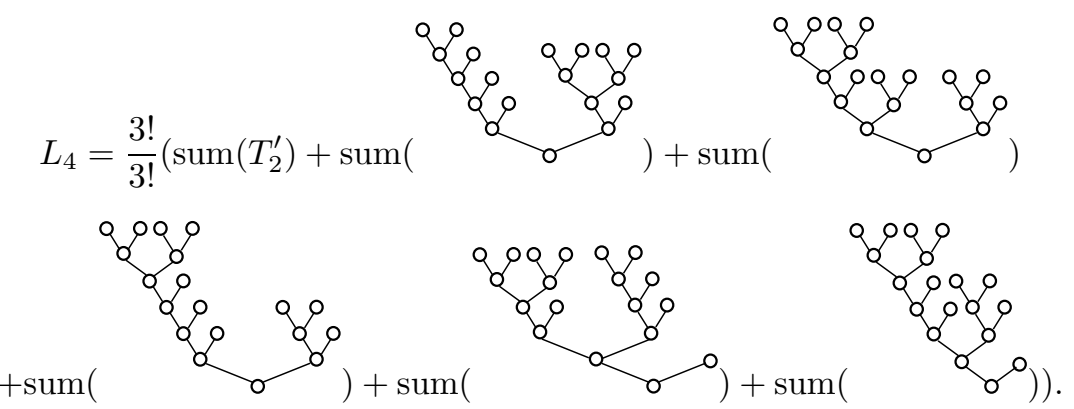

THE ElECTRONic JOURNAL OF COMBINATORICS 8 (2001), \#R2 
Hence
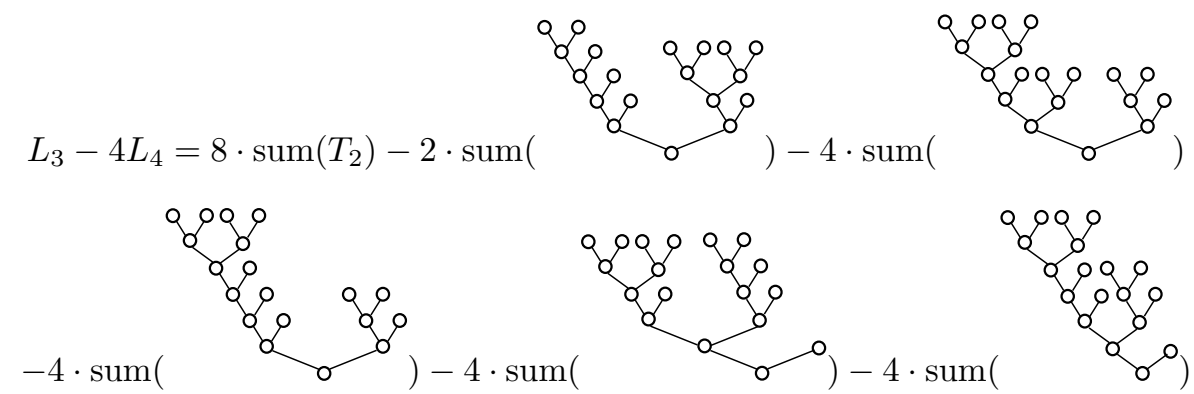

is a linear combination with leading term $T_{2}$ and is in the span of all 3-good combinations. This completes the proof of Theorem 5.3.

\section{Conclusion}

We have seen that combinatorial properties of Catalan trees translate into algebraic properties of the formal power series inverse of a system of polynomials. In particular, formal sums over equivalence classes of binary trees having a sufficiently large number of vertices can be expressed in terms of 3-good combinations, and this gives rise to the conclusion that weighted sums over equivalence classes of trees are equal to zero when certain algebraic conditions on the weight function are met. An advantage to working with trees is that we can ignore the nature of the weights. On the other hand, perhaps we omit vital information when we do so.

Since the Jacobian conjecture is true for quadratic systems of polynomials, it seems worthwhile to pursue a combinatorial proof of this fact using binary Catalan trees. One conjecture we can make is

Conjecture 6.1. The set of linear combinations of the form

$$
\operatorname{sum}(Q, u)\left(\sum_{\substack{(R, v) \in C H_{1^{k}} \circ M \\(Q, u)(R, v) S \in \mathcal{B}_{p}}}(R, v)\right) \operatorname{sum}(S)
$$

spans the set

$$
\left\{\operatorname{sum}(T): T \in \mathcal{B}_{p}\right\}
$$

whenever $p \geq f(k)(f(k)$ to be determined $)$.

We have numerical evidence to suggest that the ratio of the number of $k$-bad standard binary trees in $\mathcal{B}_{p}$ to the total number of standard trees in $\mathcal{B}_{p}$ decreases 
rapidly for fixed $k$ as $p$ increases. Perhaps there is a way to characterize all $k$ bad trees as we have in Lemma 5.8, and to prove the conjecture along the lines of Theorem 5.3.

Another way to proceed is as follows: assume the set $\mathcal{L}_{p}^{(k)}$ of linear combinations generated by binary $k$-good trees having $p$ external vertices spans the set $\left\{\operatorname{sum}(T): T \in \mathcal{B}_{p}\right\}$ when $p \geq f(k)$. Since it is easily verified that the span of the set $\mathcal{L}_{p}^{(k)}$ contains the set $\mathcal{L}_{p}^{(k+1)}$, in order to show that $\mathcal{L}_{p}^{(k+1)}$ spans the set $\left\{\operatorname{sum}(T): T \in \mathcal{B}_{p}\right\}$ when $p \geq f(k+1)$, one need only show that the span of the set $\mathcal{L}_{p}^{(k+1)}$ contains the set $\mathcal{L}_{p}^{(k)}$.

A third approach is to obtain a complete characterization of sets of unmarked binary trees whose marked counterparts share a given set of branch multisets. This would give us information we could use in order to perform Gaussian elimination on $k$-good combinations of trees, without having to resort to ad hoc arguments. We have made progress along these lines, and have discovered some interesting combinatorial algorithms along the way.

However one may choose to proceed, the investigation is bound to generate further insights into Catalan trees and, perhaps, the limits of purely combinatorial reasoning with respect to the Jacobian conjecture.

\section{References}

[1] H. Bass, E. H. Connell and D. Wright, The Jacobian conjecture: reduction of degree and formal expansion of the inverse, Bull. Amer. Math. Soc. 7 (2) (1982), 287-330.

[2] C. Cheng, T. Sakkalis, S. Wang, A case of the Jacobian conjecture, J. Pure Appl. Algebra 96 (1994), 15-18.

[3] S. Oda, The Jacobian problem and the simply-connectedness of $\mathbf{A}^{n}$ over a field $k$ of characteristic zero, Osaka University, preprint, 1980.

[4] S. Wang, A Jacobian criterion for separability, J. Algebra 65 (1980), 453494.

[5] D. Wright, Formal inverse expansion and the Jacobian conjecture, J. Pure Appl. Algebra 48 (1987), 199-219. 